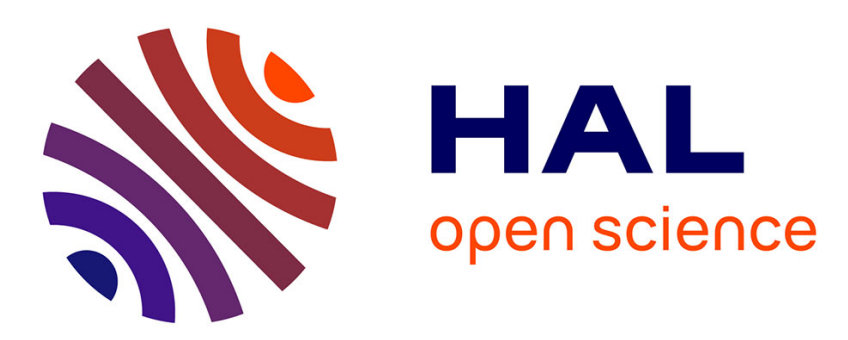

\title{
Dielectric study of the interplay between charge carriers and electron energy losses in reduced titanium dioxide
}

\author{
Rémi Lazzari, Jingfeng Li, Jacques Jupille
}

\section{To cite this version:}

Rémi Lazzari, Jingfeng Li, Jacques Jupille. Dielectric study of the interplay between charge carriers and electron energy losses in reduced titanium dioxide. Physical Review B: Condensed Matter and Materials Physics (1998-2015), 2018, 98 (7), pp.Phys. Rev. B 98, 075432. 10.1103/PhysRevB.98.075432 . hal-01872526

\section{HAL Id: hal-01872526 \\ https://hal.sorbonne-universite.fr/hal-01872526}

Submitted on 12 Sep 2018

HAL is a multi-disciplinary open access archive for the deposit and dissemination of scientific research documents, whether they are published or not. The documents may come from teaching and research institutions in France or abroad, or from public or private research centers.
L'archive ouverte pluridisciplinaire $\mathbf{H A L}$, est destinée au dépôt et à la diffusion de documents scientifiques de niveau recherche, publiés ou non, émanant des établissements d'enseignement et de recherche français ou étrangers, des laboratoires publics ou privés. 


\title{
A dielectric study of the interplay between charge carriers and electron energy losses in reduced titanium dioxide
}

\author{
Rémi Lazzari,* Jingfeng Li, and Jacques Jupille \\ CNRS, Sorbonne Université, Institut des NanoSciences de Paris, \\ UMR 7588, 4 Place Jussieu, F-75005 Paris, France
}

(Dated: August 31, 2018)

\begin{abstract}
The transport mechanism in titanium dioxide through polarons is an open issue. High Resolution Electron Energy Loss Spectroscopy (HREELS) is in principle of great relevance in such context, provided the fingerprints on the loss spectrum of the charge carriers involved in the material are disclosed. The present paper aims at evidencing those fingerprints. Through a suitable parametrization of the dielectric function, a theoretical analysis of EELS excitations in defective $\mathrm{TiO}_{2}$ rutile is developed in the framework of the semi-classical dielectric theory. The focus is put on the interplay between phonons, interband transitions and defect related excitations, namely plasmon and band gap states. Transport properties are demonstrated to be more efficiently grasped through the screening they induce on phonons than through the existence of a defined surface plasmon peak. While the corresponding imaginary part of the dielectric function only yields a slight broadening and temperature dependence of the quasi-elastic peak due to the large static dielectric function and electron effective mass, a sizable upward shift in energy and a decrease in intensity of phonons due to the real part are predicted. Band gap states also screen phonons but with downward shift in energy loss. Due to its large oscillator strength, the high energy lying surface phonon at $95 \mathrm{meV}$ is a very sensitive reporter of the combined effects of transport behavior and band gap states. Finally, it is highlighted that extracting quantitative information out of EELS experiments requires an accurate modeling of the depth profile.
\end{abstract}

\section{INTRODUCTION}

The behavior of the excess electrons present in titania and their link with the (photo)catalytic properties of the oxide have triggered a substantial amount of fundamental studies ${ }^{1-9}$, in particular at the prototypical (110) surface of rutile. Created by photon adsorption, doping or through intrinsic stoichiometric defects such as oxygen vacancies or titanium interstitials, they formally correspond to a change of oxidation state from $\mathrm{Ti}^{4+}$ to $\mathrm{Ti}^{3+}$ by populating states derived from the conduction band. Due to the large static dielectric function of $\mathrm{TiO}_{2}$ rutile ${ }^{10}$, they are accompanied by a strong lattice polaronic distorsion that screen them giving rise to specific fingerprints depending on the way they are excited ${ }^{11-13}$. On the one hand, excess electrons appear as band gap states (BGS) if the lifetime of the excitation is shorter than the phononic relaxation. In reduced $\mathrm{TiO}_{2}$, BGS related to defects ${ }^{1-8}$ are evidenced by (i) a photoemission line at $0.8-1 \mathrm{eV}$ below $E_{F}{ }^{14-17}$ that reverberates in Auger transitions involving the valence band ${ }^{18}$, (ii) an electron energy loss of $\sim 1 \mathrm{eV}^{19-22}$, (iii) features in scanning tunneling spectroscopy $^{23,24}$ or (iv) near-infrared absorption ${ }^{25,26}$ and electron spin-resonance band ${ }^{27}$. On the other hand, transport measurements ${ }^{28-31}$ favor a conduction mechanism through polarons the radius of which remains an open issue ${ }^{32}$. According to simulations, large polaron or free-like carriers in the conduction band seems to compete with more localized polaronic configurations in $\mathrm{TiO}_{2}$ rutile $24,33,34$. Up to now, the dual character of excess electrons has never been evidenced within the same experiment, not to speak about the possible coex- istence of trapped and free states that would account for transport and spectroscopic measurements.

High-Resolution Electron Energy Loss (HREELS) ${ }^{35,36}$ deserves attention in such context. Aside vibrational analysis of adsorbates, HREELS has proved its capabilities to probe solid state excitations in the surface region of materials since the birth of the technique ${ }^{37}$. Loss fingerprints of phonons, plasmons, band-to-band transitions and gap states have been accounted for in the dipolar scattering regime ${ }^{35,38}$. Numerous studies have already shown the sensitivity to surface plasmon, in particular in space-charge layers at the surface of semi-conductors, and to the coupling of those excitations to phonons as in $\mathrm{ZnO}(0001)^{39-42}, \mathrm{Si}(111)^{43-45}$, $\operatorname{InSb}(110)^{46,47}, \operatorname{InAs}(110)^{48}$ and $\operatorname{GaAs}(100)^{49-51}$. In most cases, physical parameters related to the profile of carrier concentration were derived by applying the dielectric theory ${ }^{35,52-58}$. Although blurred by multiple excitations and by a complex in-depth sensitivity, HREELS intrinsically probes dielectric properties in a way similar to optics with the advantage of covering a wide range of energy at once. The earlier HREELS experiments that were performed in the eighties on rutile $^{19,20,59}$ and anatase ${ }^{60}$ addressed the question of phonon spectra and pinpointed the existence of a defectrelated band gap feature. Since then, several groups have explored the defect reactivity with probe molecules $\left(\mathrm{H}_{2} \mathrm{O}^{61}, \mathrm{O}_{2}{ }^{62-64}, \mathrm{CO}^{65}, \mathrm{HCOOH}^{42}\right.$ etc. . $)$ by looking at (i) either the evolution of the corresponding stretching frequencies (ii) or the variation of the BGS intensity. In line with the infrared study of Baumard and Gervais ${ }^{66,67}$, HREELS experiments ${ }^{19,20,59}$ have stressed the existence 
of a relationship between the defect-related BGS and the conductivity although they pointed to intrinsic limitations of the HREELS technique that prevented in depth exploration. Rocker et al. ${ }^{19}$ suspected that "the modification in intensity and frequency of Fuchs-Kliewer phonons may be correlated with concentration of free electrons near the surface"; but they "could not resolve plasmon excitations at low energies due to the linewidth of the instrument and the background of phonons near the elastic peak". Later on, Eriksen and Edgell ${ }^{20,59}$ focussed only on the "downward shift and attenuation of the highest energy phonon loss that was attributed to modification of the effective background dielectric constant by defect excitations (BGS)". In association with Cox and Flavell ${ }^{59}$, the previous authors even tried to simulate the phonon spectra within the dielectric theory; but they were "unable to reproduce the observed changes of intensity without introducing an unduly large downward shift in phonon energy". The lack of a solution to overcome these limitations likely explains that since that time and despite the burst of interest in charge localization in $\mathrm{TiO}_{2}$ (see reviews Refs. 2-9), the correlation between the defect related BGS and the conductivity has no longer been addressed by the HREELS technique. While the n-type conductivity of reduced $\mathrm{TiO}_{2-x}$ is used to perform measurements, the existence of collective excitations due to carriers in EELS has never been disclosed although the sizeable carrier concentration obtained by reduction ${ }^{31}$ should lead to an EELS signature as in the case of more conventional semiconductors ${ }^{35}$.

To relaunch the debate, the present work aims at analyzing theoretically the capability of HREELS in correlating transport and BGS. This article is built as follows. After a reminder of the used dielectric theory of EELS (Sect. II), a suitable dielectric function including all the solid-state excitations in reduced $\mathrm{TiO}_{2}$ from infrared to ultraviolet is proposed (Sect. III). Then the impact of carrier absorptions and band gap states on the quasi-elastic peak (Sect. IV A) and the phonon excitation (Sects. IV B-IV C) is analyzed theoretically by stressing on the effect of screening. Beyond the exploration of specific fingerprints for the various excitations that exist in the surface region of the oxide, new prospects are opened on interplays between those excitations. The role of the relative probing depth as a function of energy loss is also developped (Sect. V).

\section{REMINDER ON DIELECTRIC THEORY OF EELS AND METHODS}

All simulations have been performed within the semi-classical dielectric theory ${ }^{35,52-58}$ the relevance of which in the modeling of EELS spectra has been proven since the early beginning of the technique ${ }^{37}$ and in depth over the years ${ }^{38,68}$.

In a EELS experiment, an electron of charge $-e$, mass $m_{e}$, wavevector $\mathbf{k}_{I}$, incident energy $E_{I}=\hbar^{2} k_{I}^{2} / 2 m_{e}$ and velocity $\mathbf{v}_{I}=\hbar \mathbf{k}_{I} / m_{e}$ impinges on the sample surface at an angle $\Theta_{I}$ before being scattered along the direction given by wavevector $\mathbf{k}_{S}$. In the following, the subscripts $\|$ and $\perp$ stand for components parallel and perpendicular to the surface, respectively. In the standard dipole scattering theory, the electron is a classical particle that follows a nearly unperturbed specular trajectory, i.e. $k_{\|}=\left|\mathbf{k}_{\|, I}-\mathbf{k}_{\|, S}\right| \ll k_{I}$, and bounces off the surface giving rise to a transient electrostatic field that produce excitations in the substrate. The single loss probability for an electron inelastically scattered in an energy windows $\hbar d \omega$ around $\hbar \omega \ll E_{I}$ and close to the specular direction reads ${ }^{35,52-58}$ :

$$
P_{c l}\left(\mathbf{k}_{I}, \omega\right)=\frac{4 e^{2}}{\hbar v_{\perp}} \int_{D} \frac{\left(k_{\|} v_{\perp}\right)^{3}}{\left[\left(\omega-\mathbf{k}_{\|} \cdot \mathbf{v}_{\|}\right)^{2}+\left(k_{\|} v_{\perp}\right)^{2}\right]^{2}} \operatorname{Im}\left[-\frac{1}{1+\xi\left(\mathbf{k}_{\|}, \omega\right)}\right] \frac{d^{2} \mathbf{k}_{\|}}{k_{\|}^{2}}=\int_{D} F\left(\mathbf{k}_{I}, \omega\right) G\left(k_{\|}, \omega\right) d^{2} \mathbf{k}_{\|}
$$

where $\hbar$ is the Planck constant divided by $2 \pi$ and $v_{\|}=v_{I} \sin \Theta_{I}, v_{\perp}=v_{I} \cos \Theta_{I}$. The $\mathbf{k}_{\|}$integration domain $D$ in Eq. 1 is defined by the incident beam divergence and detector angular acceptance, the so-called slit integration ${ }^{35}$. To avoid the cumbersome ${ }^{69}$ underlying fourth integral over incoming/scattering angles for convergent/divergent beams, analyses have been performed with an equivalent circular detector acceptance $\theta_{c}$ and a parallel incident beam. In that case, $\mathbf{k}_{\|}$depends on $\theta_{S}$ $\left(0<\theta_{S}<\theta_{c}\right)$, the small angle from the specular direction and on $\phi_{S}$, the azimuth angle looking downwards the origin 22,35 ,

$$
\mathbf{k}_{\|}=k_{I}\left\{\begin{array}{l}
-\theta_{S} \cos \phi_{S} \cos \Theta_{I}+\theta_{E} \sin \Theta_{I} \\
-\theta_{S} \sin \phi_{S}
\end{array}\right.
$$

with $\theta_{E}=\hbar \omega / 2 E_{I}$. If the integrand in Eq. 1 depends only on the modulus of $k_{\|}$, the integration over $\left(\theta_{S}, \phi_{S}\right)$ in Eq. 1 can be transformed into a one-dimensional radial integral in the $\mathbf{k}_{\|}$plane (see Appendix of Ref. 58 for details), a process that speed up calculations. $P_{c l}\left(\mathbf{k}_{I}, \omega\right)$ involves two terms. On the one hand, the kinematical prefactor or sensitivity function $F\left(\mathbf{k}_{I}, \omega\right)$ gives rise to the angular behavior of the EELS cross section with an intense lobe of aperture $\theta_{E}$ close to the specular 
direction $^{35}$ and a fast decay with $\hbar \omega$. On the other hand, the loss function $G\left(k_{\|}, \omega\right)$ depends on the effective dielectric function $\xi\left(\mathbf{k}_{\|}, \omega\right)$ of the probed interface which, for a homogeneous isotropic non-dispersive semi-infinite substrate is nothing else than the dielectric function of the medium $\epsilon(\omega)$. Indeed, since the present analyses have been mainly performed in the specular direction, no intrinsic $\mathbf{k}_{\|}$-dependence (i.e. dispersive behavior of the dielectric function ${ }^{70}$ ) has been accounted for. For an uniaxial material like $\mathrm{TiO}_{2}, \xi\left(\mathbf{k}_{\|}, \omega\right)$ depends in a complex way on the relative orientation of the crystallographic axis and of the scattering plane ${ }^{57}$ (see Sect. S1 of Supplemental Material ${ }^{71}$ ). Hopefully, when the c-axis (or [001]) of the quadratic structure is perpendicular (respectively, parallel) to the scattering plane, $\xi\left(\mathbf{k}_{\|}, \omega\right) \simeq \epsilon_{\perp}^{a}(\omega)\left(\right.$ respectively, $\left.\xi\left(\mathbf{k}_{\|}, \omega\right) \simeq \sqrt{\epsilon_{\perp}^{a}(\omega) \epsilon_{\|}^{c}(\omega)}\right)$ becomes $\mathbf{k}_{\|}$-independent. It is given by the two components of the dielectric tensor $\epsilon_{\|}^{c}(\omega)$ and $\epsilon_{\perp}^{a}(\omega)$ along directions parallel and perpendicular to the c-axis ${ }^{72}$. As detailed in Sect. S1 of Supplemental Material ${ }^{71}$, Eq. 1 can be generalized to account recursively for any profile of dielectric function along the normal to the surface ${ }^{58}$.

When the coupling between the incident electron and the medium is relatively strong or when the energy loss is of the same order of magnitude as $k T$ as in the case of surface plasmons, the single scattering probability Eq. 1 breaks down. The spectrum involves a complex combination of intense multiple energy losses and gains due to the creation and annihilation of quanta of excitations ${ }^{37}$. The problem at hand can be treated completely (i) on quantum-mechanical point of view either in the first Born approximation or in a more complex way including multiple scattering ${ }^{54,55}$ or (ii) through in a semi-classical method by considering the electron as a classical source of perturbation for the quantized boson field of surface excitations ${ }^{35,52,70}$. Within this more tractable approach, the HREELS spectrum $P(\omega)$ at finite temperature $T$ including multiple excitations is generated through:

$$
\begin{aligned}
P(\omega) & =\frac{1}{2 \pi} \int e^{-i \omega t} \mathcal{R}(t) e^{[\mathcal{P}(t)-\mathcal{P}(0)]} \\
& =e^{-\mathcal{P}(0)} R(\omega) \otimes\left\{\delta(\omega)+\sum_{n=1}^{\infty} \frac{1}{n !}[\mathcal{P}(\omega)]^{n \otimes}\right\}, \\
\mathcal{P}(t) & =\int_{0}^{+\infty} d \omega^{\prime} P_{c l}\left(\omega^{\prime}\right) \\
& \times\left[\left(n_{\omega^{\prime}}+1\right)\left(e^{i \omega^{\prime} t}+1\right)+n_{\omega^{\prime}}\left(e^{-i \omega^{\prime} t}-1\right)\right],
\end{aligned}
$$

where $n_{\omega}=\left[e^{\hbar \omega / k_{B} T}-1\right]^{-1}$ is the Bose-Einstein distribution, $k_{B}$ the Boltzmann constant, $\otimes$ the convolution product. $\mathcal{P}(t)$ and $P(\omega)$ are related by Fourier transform. In order to simulate a finite resolution of the spectrometer by a convolution, an instrumental transfer function $\mathcal{R}(t)=\int e^{-i \omega t} \mathcal{R}(\omega) d \omega$ can be easily introduced before the back Fourier transform in Eq. 3. The EELS spectrum $P(\omega)$ appears as the multiple convolution (Eq. 4) of the single gain-loss function $\mathcal{P}(\omega)$ which generates not only multiple losses from a given transition but also combination features between different transitions and also gain peaks at finite temperature. The series of multiple losses follows a Poisson statistics (Eq. 4) $)^{37,73}$. Finally, the $\mathbf{k}_{\|}$integration over domain $D$ has been restricted herein to one-quantum loss processes i.e. performed on $P_{c l}\left(\mathbf{k}_{I}, \omega\right)($ Eq. 1) and not on the multiple excitation cross section $P(\omega)$ (Eq. 3) as it should $\mathrm{be}^{70}$. But, the underlying approximation is usually hidden in the experimental uncertainties ${ }^{70}$.

On a practical point of view, once the function $\xi\left(\mathbf{k}_{\|}, \omega\right)$ is defined from the profile of the dielectric function (see Sect. S1 of Supplemental Material ${ }^{71}$ ), $P_{c l}\left(\Theta_{I}, E_{I}, \omega\right)$ (Eq. 1) is obtained by $1 \mathrm{D}$ auto-adaptative numerical integration $^{74}$ under the symplifying assumption of a circular detection slit ${ }^{58}$. It is then Fourier transformed after multiplication by the required Bose-Einstein statistics (Eq. 6) for the selected temperature $T$ to obtain $\mathcal{P}(t)$ (Eq. 5). The exponential of this latter is then multiplied by the Fourier transform of the apparatus point spread function $\mathcal{R}(t)$ before being back Fourier transform to obtain the final simulated HREELS spectrum $P(\omega)$. In passing, a great care has been taken in the sampling in the integrals in Eqs. 1,5-6 which may be biased by numerical issues at low plasmon energies or dampings. Fast Fourier transform was used with an energy step down to $\Delta \hbar \omega=10^{-4} \mathrm{meV}$. If not stated afterward, the following simulations parameters corresponding to experiments $^{22,69}$ have been used throughout this theoretical study: beam energy $E_{I}=8 \mathrm{eV}$ or $E_{I}=38 \mathrm{eV}$; incident angle $\Theta_{I}=60^{\circ}$; circular aperture in detection $\theta_{c}=1^{\circ}$; gaussian shape for the apparatus resolution function ${ }^{75}$ $\mathcal{R}(\omega)=\frac{1}{\sigma_{a} \sqrt{2 \pi}} \exp \left[-\frac{\hbar^{2} \omega^{2}}{2 \sigma_{a}^{2}}\right]$ with a Full-Width at HalfMaximum (FWHM) of $\Delta_{a}=2 \sqrt{2 \ln (2)} \sigma_{a}=8 \mathrm{meV}$ for HREELS at $E_{I}=8 \mathrm{eV}$ or $\Delta_{a}=50 \mathrm{meV}$ for EELS at $E_{I}=38 \mathrm{eV}$; temperature $T=300 \mathrm{~K}$. Simulations have been performed with a beam perpendicular to the c-axis i.e. with a dominant contribution from $\epsilon_{\perp}^{a}(\omega)$. If not specified, simulations involve hereafter only a semiinfinite substrate; profiles of dielectric function treated in Sect. V have been sliced in a staircase way to compute recursively $\xi\left(\mathbf{k}_{\|}, \omega\right)$ until reaching convergence of the simulated spectrum.

\section{THE DIELECTRIC FUNCTION OF $\mathrm{TIO}_{2}$ FROM INFRARED TO ULTRAVIOLET}

An accurate expression of the two components $\epsilon_{\|}^{c}(\omega)$ and $\epsilon_{\perp}^{a}(\omega)$ of the dielectric tensor of $\mathrm{TiO}_{2}$ is required to model the energy loss spectrum of $\mathrm{TiO}_{2}$ over a large spectral range (up to $6 \mathrm{eV}$ ). To obtain a tractable analytic expression of the dielectric function, $\epsilon_{\|}^{c}(\omega)$ and $\epsilon_{\perp}^{a}(\omega)$ have been decomposed into contributions due to phonon $\epsilon_{P h}(\omega)$, band-to-band transitions $\epsilon_{I b}(\omega)$, band 
gap states $\epsilon_{g s}(\omega)$ and Drude or plasmon-like excitations $\epsilon_{P l}(\omega)$ (Fig. 1):

$$
\epsilon_{T_{i O} O_{2}}(\omega)=\epsilon_{P h}(\omega)+\epsilon_{I b}(\omega)+\epsilon_{g s}(\omega)+\epsilon_{P l}(\omega) .
$$

As seen in what follows, the two former components of the equation can be extracted from tabulated data. However, new approaches are required to determine the contribution $\epsilon_{g s}(\omega)+\epsilon_{P l}(\omega)$. If not stated specifically, the labels $\|, \perp$ for the dielectric function are assumed to be implicit hereafter. Each term will be detailed one after the other. Only dipolar EELS simulations will be carried out $\left(k_{\|} \simeq 0\right)$. Therefore, any dependence on wavevector transfer parallel to the surface $k_{\|}$, i.e. non local effects, can be safely discarded.

\section{A. Phonons}

As suggested in the infrared reflectivity analysis of Gervais and Piriou ${ }^{67,77,78}$ and in the accurate midinfrared and far-infrared ellipsometric measurements of Schöche et al. ${ }^{10}$, the contribution of infrared active optical phonons is described through a factorized model:

$$
\epsilon_{P h}(\omega)=\epsilon_{P h, \infty} \prod_{n} \frac{\omega_{L O, n}^{2}-\omega^{2}-i \Gamma_{L O, n} \omega}{\omega_{T O, n}^{2}-\omega^{2}-i \Gamma_{T O, n} \omega} .
$$

$\omega_{L O}, \omega_{T O}$ and $\Gamma_{L O}, \Gamma_{T O}$ stand for the frequencies and dampings of the longitudinal and transverse phonon modes at the center of the Brillouin zone. $\epsilon_{P h, \infty}$ is the optical dielectric function i.e. at frequencies much higher than phonons. Parameters from Ref. 10 given in Tab. S1 of Supplemental Material ${ }^{71}$ match perfectly with all previous determinations of phonon modes in rutile via infrared probe ${ }^{77,79}$, neutron scattering ${ }^{80}$ and $a b$ initio modelling ${ }^{81-83}$. This infrared ellipsometry analysis was performed on stoichiometric $\mathrm{TiO}_{2}$ samples ${ }^{84}$ of various orientations (001), (110) and $(111)^{10}$. The group theory decomposition in irreducible representation of $\mathrm{TiO}_{2}$ rutile phonons ${ }^{20,80,81,85}$ (point group $D_{4 h}^{1}$ or $P 4 / \mathrm{mmm}$ ): $\Gamma=A_{1 g}+A_{2 g}+A_{2 u}+B_{1 g}+B_{2 g}+2 B_{1 u}+E_{g}+3 E_{u}$ shows that only four modes $\left(A_{2 u}+3 E_{u}\right)$ have infrared (EELS) dipole activity with longitudinal/transverse splitting. $E_{u}$ modes are doubly degenerated. Therefore, the summation in Eq. 8 extends over $n_{\perp}=3 E_{u}$ and $n_{\|}=1 A_{2 u}$ modes. The factorized form of $\epsilon(\omega)$ was preferred over the classical summation of damped oscillators $^{20,79}$ to allow independent broadenings of transverse and longitudinal phonons and asymmetric shapes as observed in ionic crystal with several modes having large TO-LO splitting ${ }^{10,77,78}$. Furthermore, it explicitly expresses the longitudinal phonon frequencies probed in transmission EELS as the poles of $1 / \epsilon_{P h}(\omega)$. In reflection EELS, Fuchs-Kliewer modes ${ }^{35,37}$ appear as the maxima of (i) $\operatorname{Im}\left[1 /\left(1+\epsilon_{\perp, P h}(\omega)\right]\right.$ at $\hbar \omega_{s p h, 1}=45.3 \mathrm{meV}$, $\hbar \omega_{s p h, 2}=54.6 \mathrm{meV}, \hbar \omega_{s p h, 3}=95.4 \mathrm{meV}$ and (ii) of $\operatorname{Im}\left[1 /\left(1+\sqrt{\epsilon_{\|, P h}(\omega) \epsilon_{\perp, P h}(\omega)}\right]\right.$ at $\hbar \omega_{s p h, 1}=45.6 \mathrm{meV}$,

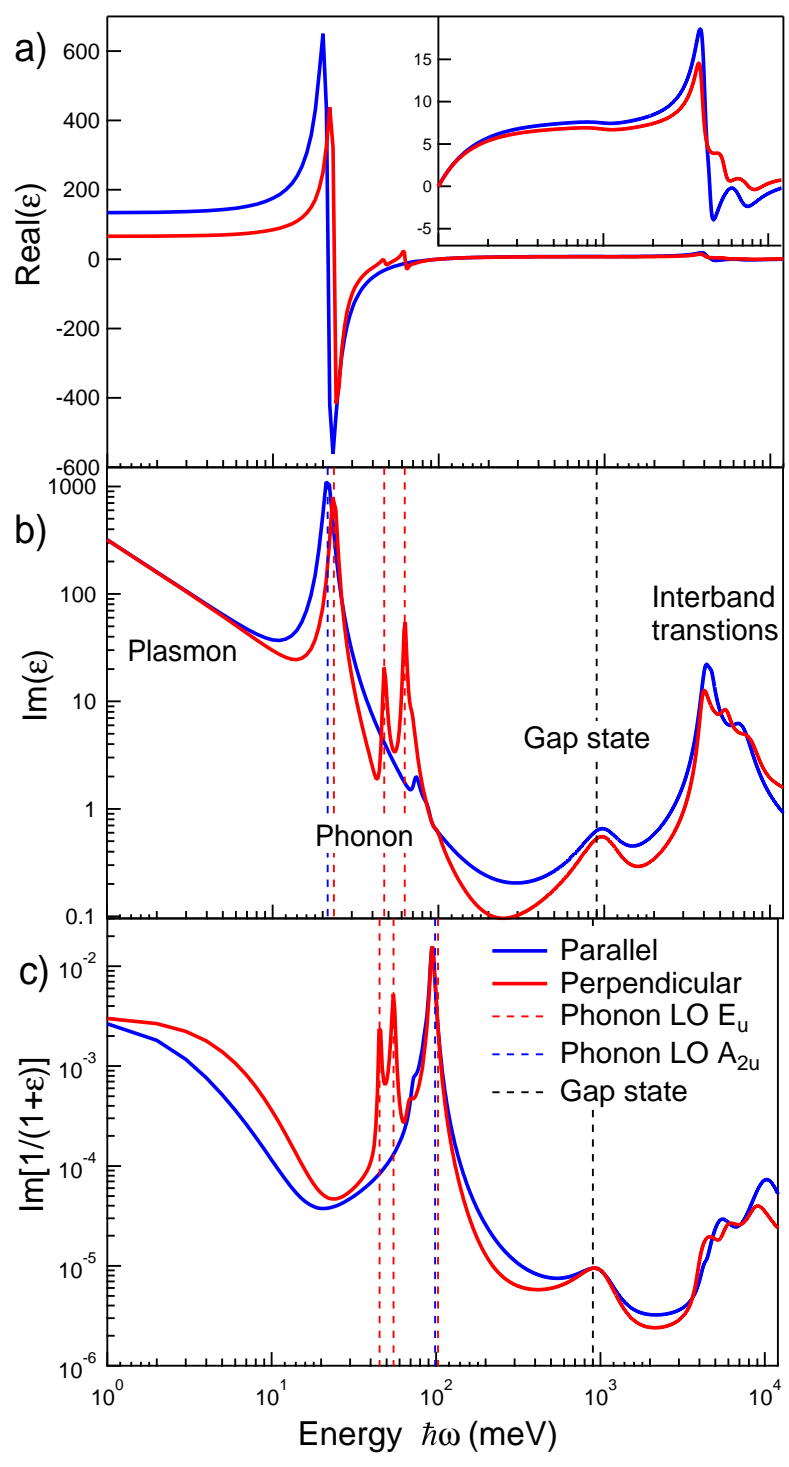

FIG. 1. a) Real and b) imaginary part of the $\mathrm{TiO}_{2}$ dielectric functions $\epsilon_{\|}^{c}(\omega)$ (blue line), $\epsilon_{\perp}^{a}(\omega)$ (red line) (Eq. 7). c) Corresponding loss function $\operatorname{Im}[1 / 1+\epsilon(\omega)]$. The inset shows a zoom of $\operatorname{Re}[\epsilon(\omega)]$ over the $0.1-12 \mathrm{eV}$ range. Vertical dotted blue and red lines pinpoint the frequencies of transverse $\omega_{T O}$ and longitudinal $\omega_{L O}$ phonons frequencies of bulk $\mathrm{TiO}_{2}$ used in $\epsilon_{P h}(\omega)$ (Eq. 8). $\hbar \omega_{p}=80 \mathrm{meV}, \hbar \Gamma_{P}=20 \mathrm{meV}$, $\hbar \omega_{g s}=1000 \mathrm{meV}, \hbar \Gamma_{g s}=600 \mathrm{meV}, \Omega_{g s}=500 \mathrm{meV}$ have been used to describe the Drude (Eq. 12) and band gap states (Eq. 10) contributions; the phonon and interband transition counterparts are extracted from Refs. 10 and 76, respectively. The black dotted line points at the position of BGS.

$\hbar \omega_{s p h, 2}=55.4 \mathrm{meV}, \hbar \omega_{s p h, 3}=93.8 \mathrm{meV}$ for incident planes perpendicular and parallel to the c-axis, respectively (Fig. S1 in Supplemental Material ${ }^{71}$ ). The similarity of the two sets of frequencies stems from the accidental degeneracy of $A_{2 u, \|}$ and $E_{u, \perp}{ }^{20,59}$. Four symmetry forbidden additional phonons of much lower 
oscillator strengths, two along each direction, have also been reported ${ }^{10,77}$ (Tab. S1 and Fig. S2 in Supplemental Material $^{71}$ ). They correspond to maxima in the phonon density of states and are probably activated by bulk defects through folding of the Brillouin zone. For completeness, they were accounted for in the present study.

The severe longitudinal/transverse splitting of phonon modes in rutile (Tab. S1 of Supplemental Material ${ }^{71}$ ) results in large values of static dielectric constants. The Lyddanne-Sachs-Teller relation ${ }^{86}$ :

$$
\epsilon(0)=\epsilon_{P h, \infty} \prod_{n} \frac{\omega_{L O, n}^{2}}{\omega_{T O, n}^{2}} .
$$

leads to $\epsilon_{\|}^{c}(0)=153$ and $\epsilon_{\perp}^{a}(0)=84.7^{10,77}$ in agreement with capacitance measurements ${ }^{87}$. Those values demonstrate the importance of the electron-phonon coupling that is at the origin of polaronic behavior of excess electrons. As a matter of comparison, $\epsilon(0)$ of $\mathrm{ZnO}$ wurtzite and $\mathrm{Si}$ are one order of magnitude lower $\left(\epsilon_{Z n O}(0)=8.7 ; \epsilon_{S i}(0)=11.7\right)$.

\section{B. Interband transitions}

The valence and conduction bands of $\mathrm{TiO}_{2}$ rutile are dominated by $\mathrm{O} s p$ and strongly localized Ti $3 d$ states, respectively with a direct $\Gamma$-point band gap of 3.1$3.2 \mathrm{eV}^{83,88,89}$ and a complex role of crystal field effect on anisotropy at large energies ${ }^{90,91}$. The UV-visible contribution $\epsilon_{I b}(\omega)$, in particular the part related to interband transitions, was extracted from the compilation of Ref. 76. For the sake of EELS simulation, it was parametrized and fitted using the critical point transition approach ${ }^{92}$ :

$$
\begin{aligned}
\epsilon_{I b}(\omega)=\epsilon_{I b, \infty} & +\sum_{m}\left\{\frac{C_{m} e^{i \Phi_{m}}}{\left(\omega_{m}-\omega-i \Gamma_{m}\right)^{\mu_{m}}}\right. \\
& \left.+\frac{C_{m} e^{-i \Phi_{m}}}{\left(\omega_{m}+\omega+i \Gamma_{m}\right)^{\mu_{m}}}\right\} .
\end{aligned}
$$

$\epsilon_{I b, \infty}, C_{m}, \Phi_{m}, \mu_{m}, \omega_{m}, \Gamma_{m}$, are the high frequency dielectric function and the amplitude, the phase, the pole order, the frequency, the damping of the $m^{\text {th }}$ critical point, respectively. Compared to the standard oscillator $^{93}$, the representation accounts for transitions between band pairs with a substantial integration over $k$-space leading to an apparent broadening larger than intrinsic linewidth to functional dependence other than simple poles. A satisfactory fit in the $0.6-12 \mathrm{eV}$ range of data of Ref. 76 (Fig. S3 in Supplemental Material ${ }^{71}$ ) was achieved with only three transition points along the parallel and perpendicular direction and zero phases (Fig. 1). Data were carefully matched to the $\epsilon_{P h}(\omega)$ value from Ref. 10.

\section{Defect induced band gap states and optical absorption}

The BGS correspond to the optical absorption by a polaronic trapping state due to defect-related excess electrons, a polaron being a quasiparticle made of an electron and the accompanying phonon cloud due to lattice distortions. The slow motion of the atoms allows to freeze the self-trapping potential well during photoemission ${ }^{12}$, which explains why thermal activation energy ${ }^{28-31}$ differs from optical transition energy for excess electrons. The line shape of absorption from polaronic states depends on the degree of electron-phonon coupling, on the temperature or even on the dimensionality of space ${ }^{94,95}$. Two extreme cases can be distinguished, large and small polaron ${ }^{11}$. The photo-ionization of the hydrogenoic ground state of the potential well of the large polaron excites electrons to a continuum of unbound states. The absorption coefficient $\alpha \sim(k R)^{3} / \omega\left[1+(k R)^{2}\right]^{4}$, where $\hbar k=\sqrt{2 m\left(\hbar \omega-3 E_{p}\right)}$ is the free-carrier wavevector and $R$ the radius of the polaron, leads to a peaked asymmetric function that starts above three times the polaron binding energy $E_{p}$. Conversely, the self-trapped carrier of the small polaron is excited from its localized state to an adjacent site. The absorption coefficient $\alpha \sim \exp \left[-\left(2 E_{b}-\hbar \omega\right)^{2} / \Delta^{2}\right] / \omega$ depends on the polaron binding energy $E_{b}$ and on the thermal broadening $\Delta$ of levels. It shows a gaussian line shape with an asymmetry on the higher energy side and a strong temperature dependence in contrast to the large polaron model.

Beside the debate on the location of excess charges in the subsurface region of $\mathrm{TiO}_{2}{ }^{17,96-99}$, little is known about their degree of localization $22,24,33,100$, i.e. the polaron radius. The large polaron model is favored in anatase $^{24,101,102}$. The polaron radius is not settled yet in rutile ${ }^{24,34,67}$, although the small polaron model is currently favored in bulk ${ }^{25,103}$ and at (110) surfaces ${ }^{104}$. The apparatus sensitivity function and the actual profile of excess electrons to which several types of defects contribute $^{22}$ makes difficult the determination of the polaron radius via the asymmetry of the BGS in EELS. Therefore, it has been chosen herein to represent BGS optical absorptions by a simple oscillator model:

$$
\epsilon_{g s}(\omega)=\frac{\Omega_{g s}^{2}}{\omega_{g s}^{2}-\omega^{2}-i \Gamma_{g s} \omega},
$$

where $\omega_{g s}, \Gamma_{g s}, \Omega_{g s}$ are the frequency, damping and strength of the oscillator, respectively. This form is supposed to grasp most of the physics of screening discussed hereafter. 


\section{Excitation due to itinerant motion of carriers: the Drude model}

Reduced rutile is indubitably a $n$-type semiconductor whose transport properties have been much studied $^{30,31,105}$. But their nature is unclear. In particular the puzzling question of the large mobility ${ }^{24,34}$ at low temperature is not understood, not speaking about the role of defects ${ }^{22}$ (oxygen vacancies and/or titanium interstitials). Is transport in titanium dioxide due to a dual behavior of excess electrons or to the coexistence of trapped and free states giving rise to BGS and conductivity ${ }^{24,34}$ ?

Because of the strong electron-phonon coupling, transport is often debated in terms of the impact of the above described small and large polarons on conductivity $^{24,34,67}$. The ability of a polaron to move coherently in an alternative field is always restricted as it is linked to the atomic motion. However, a polaron can have a Drude-like carrier absorption ${ }^{11,94,95}$ at low frequency if it moves itinerantly with a mean free path greater than the inter-site separation. For a large polaron, the Drude-like behavior occurs only at frequencies below the characteristic phonon frequency involved in the trapping. For a small polaron, the condition is more stringent. The extremely narrow bands that characterize small polaronic carriers may preclude itinerant (coherent) small-polaron motion in all but the most idealized conditions ${ }^{11}$.

To account for EELS findings ${ }^{69}$, the carrier excitations and therefore the conductivity at low frequency are described in this work through a classical Drude formula:

$$
\epsilon_{P l}(\omega)=-\frac{\omega_{P}^{2}}{\omega^{2}+i \omega \Gamma_{P}} \quad \text { with } \quad \omega_{P}^{2}=\frac{n e^{2}}{m_{e} m^{*} \epsilon_{0}},
$$

where the plasma frequency $\omega_{P}$ is given in terms of the carrier density $n$ and their effective polaronic mass $m^{*},\left(e, m_{e}, \epsilon_{0}\right)$ being respectively the elementary electric charge, the electron rest mass and the vacuum permittivity. The damping $\Gamma_{P}$ is related to the carrier mobility $\mu$ through $\Gamma_{P}=2 \pi e /\left(m_{e} m^{*} \mu\right)$. The Drude model is the ground level of description of the frequency dependent (or optical) conductivity $\sigma(\omega)\left(\epsilon(\omega)=\epsilon_{0}+i \sigma(\omega) / \omega\right)$ through a collision relaxation time ${ }^{86}$. Baumard and Gervais $^{66,67}$ demonstrated that this basic model correctly fits the infrared reflectivity behavior in heavily reduced samples $\mathrm{TiO}_{2-x}(x \simeq 0.001-0.05)$ over a large frequency range $(25-375 \mathrm{meV})$ well above the transverse optical frequency of phonons. They found that plasmons couple to longitudinal phonon modes and that $\omega_{P}^{2}$ depends linearly on reduction level $x$. Through temperature dependent measurements, they concluded that the activation of carriers to the conduction band constitutes the essential feature of the electrical conductivity. Frequencydependent conductivity below the terahertz $(4 \mathrm{meV})$ were also successfully interpreted with the Drude approach for optically created carriers ${ }^{32,106}$ as well as for hydrogen doping ${ }^{107}$ at low temperature. By using the Drude model, Hendry et $a l .{ }^{32,106}$ concluded that transport occurs through a polaron in the intermediate regime of coupling, neither large nor small with a strong anisotropy of effective mass and electron mobility.

\section{THE INTERPLAY BETWEEN EXCITATIONS IN REDUCED TIO $_{2}$}

The energy losses that stem from carriers-related collective excitations have never been disclosed, although a fingerprint of conductivity is to be expected. The case is now explored by simulating (HR)EELS spectra of $\mathrm{TiO}_{2}(110)$ at low and large impact energies on the basis of the above described dielectric function. The main features of such spectra are exemplified in Fig. 2. Beside the single phonon excitations $\omega_{s p h, 1}, \omega_{s p h, 2}, \omega_{s p h, 3}$ already present in the loss function (Fig. 1-c and Fig. S2 of Supplemental Material), multiple and combination excitations are accounted for as well as gain peaks on the negative energy loss axis. Due to the sensitivity function $F\left(\mathbf{k}_{I}, \omega\right)$ (Sect. II), intensities of the elastic peak and phonon losses are several orders of magnitude higher than that of interband-transitions located above $3.2 \mathrm{eV}$ (inset of Fig. 2). Gap states appear clearly as an isolated broad feature in the EELS spectrum of reduced $\mathrm{TiO}_{2}{ }^{20-22}$. In the following, through dielectric simula-

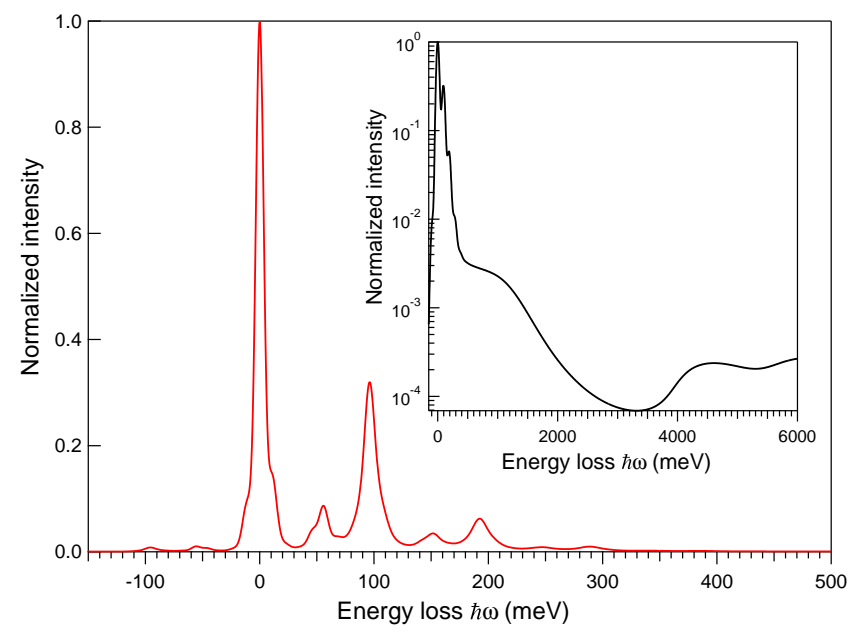

FIG. 2. An example of simulated HREELS spectrum in the region of phonons $\left(E_{I}=8 \mathrm{eV}\right.$ and $\left.\Delta_{a}=8 \mathrm{meV}\right)$ and of the interband transitions (inset; $E_{I}=38 \mathrm{eV}$ and $\Delta_{a}=50 \mathrm{meV}$ ). The beam is perpendicular to the c-axis for which the effective dielectric function amounts only to $\xi(\omega)=\epsilon_{\perp}^{a}(\omega)$ (Sect. II). Due to a fortuitous degeneracy of phonon modes ${ }^{20,59}$, the crystal orientation at $90^{\circ}$ leads to a similar spectrum (see Sect. S2 of Supplemental Material $\left.{ }^{71}\right)$. Calculations include plasmon $\left(\hbar \omega_{P}=120 \mathrm{meV}, \hbar \Gamma_{P}=2 \mathrm{meV}\right)$ and BGS $\left(\hbar \Omega_{g s}=\right.$ $1000 \mathrm{meV}, \hbar \omega_{g s}=1000 \mathrm{meV}, \hbar \Gamma_{g s}=600 \mathrm{meV}$ ) excitations.

tions with typical experimental parameters, this section 
aims to identify the EELS signatures of the various excitations of excess electrons, including the interplay of the surface plasmons with the quasi-elastic peak and the screening of phonons by either carrier excitations or band gap states.

\section{A. Quasi-elastic peak broadening and surface plasmons}

When low frequency conductivity is described only through a Drude term $\epsilon(\omega)=\epsilon(0)-\omega_{P}^{2} /\left(\omega^{2}+i \omega \Gamma_{P}\right)$, the classical loss function which reads:

$G(\omega)=\operatorname{Im}\left[-\frac{1}{1+\epsilon_{P l}(\omega)}\right]=\frac{1}{1+\epsilon(0)} \frac{\Gamma_{P} \omega_{s p}^{2} \omega}{\left[\omega_{s p}^{2}-\omega^{2}\right]^{2}+\Gamma_{P}^{2} \omega^{2}}$

has a nearly lorentzian shape that peaks at the so-called surface plasmon frequency $\omega_{s p}=\omega_{P} / \sqrt{1+\epsilon(0)}$. These excitations are coherent delocalized electron oscillations that exist at the interface between materials (vacuum/solid here). They are the electronic equivalent of surface phonons ${ }^{35,108}$. For doped semiconductors, the conductivity manifests itself in EELS either as a isolated peak or as a broadening of the elastic peak due to multiple $\omega_{s p}$-excitations ${ }^{35,43}$. The coupling to the incident electrons may be so intense that the peak is better named "quasi-elastic" as nearly all reflected electrons suffer from energy loss. As an incipient ferroelectric compound, $\mathrm{TiO}_{2}$ possesses a much higher static dielectric function $\epsilon(0)\left(\epsilon_{\|}^{c}(0)=153, \epsilon_{\perp}^{a}(0)=84.7\right.$, see Sect. III A) than $\mathrm{ZnO}$ and $\mathrm{Si}\left(\epsilon_{Z n O}(0)=8.7\right.$, $\left.\epsilon_{S i}(0)=11.7\right)$ of which surface plasmon excitation and accumulation/depletion layers have been studied by EELS $^{40-51}$. In addition, the polaronic nature of the electronic transport leads to a much higher effective mass of $m^{*}=8-10^{31,32,67}$ than the estimate by $a b$ initio band-structure calculations for a rigid lattice $\left(m_{\perp}^{*}=1.1, m_{\perp}^{*}=0.57^{83}\right)$. The combination of the two effects yields a $\omega_{s p} \sim 1 / \sqrt{m^{*}} \sqrt{1+\epsilon(0)}$-value two orders of magnitude lower than for classical semiconductors at a given carrier concentration $n$ (see top scales of Fig. 4). Consequently, the limited resolution makes difficult the direct EELS detection of surface plasmon in $\mathrm{TiO}_{2}$. Only a broadening of the quasi-elastic peak is expected.

Full numerical simulations of the elastic peak shape have therefore been carried out herein to identify both the effect of carrier concentration $n / m^{*}$ and damping $\Gamma_{P}$ on the FWHM of the quasi-elastic peak of rutile (Fig. 3). Besides instrumental broadening $\Delta_{a}$, the FWHM is driven by the superposition of multiple excitations of surface plasmons $\omega_{s p}$, which are better visible when $\Gamma_{P}$ and $\Delta_{a}$ values are artificially reduced (Fig. 3-b). Their intensities follow a Poisson distribution (Eq. 3) modulated by the Bose-Einstein statistics (Eq. 6). As predicted by the analytic formula of Ref. 43 (dotted line of Fig. 4, Sect. S3 of Supplemental Material ${ }^{71}$ ), increas-

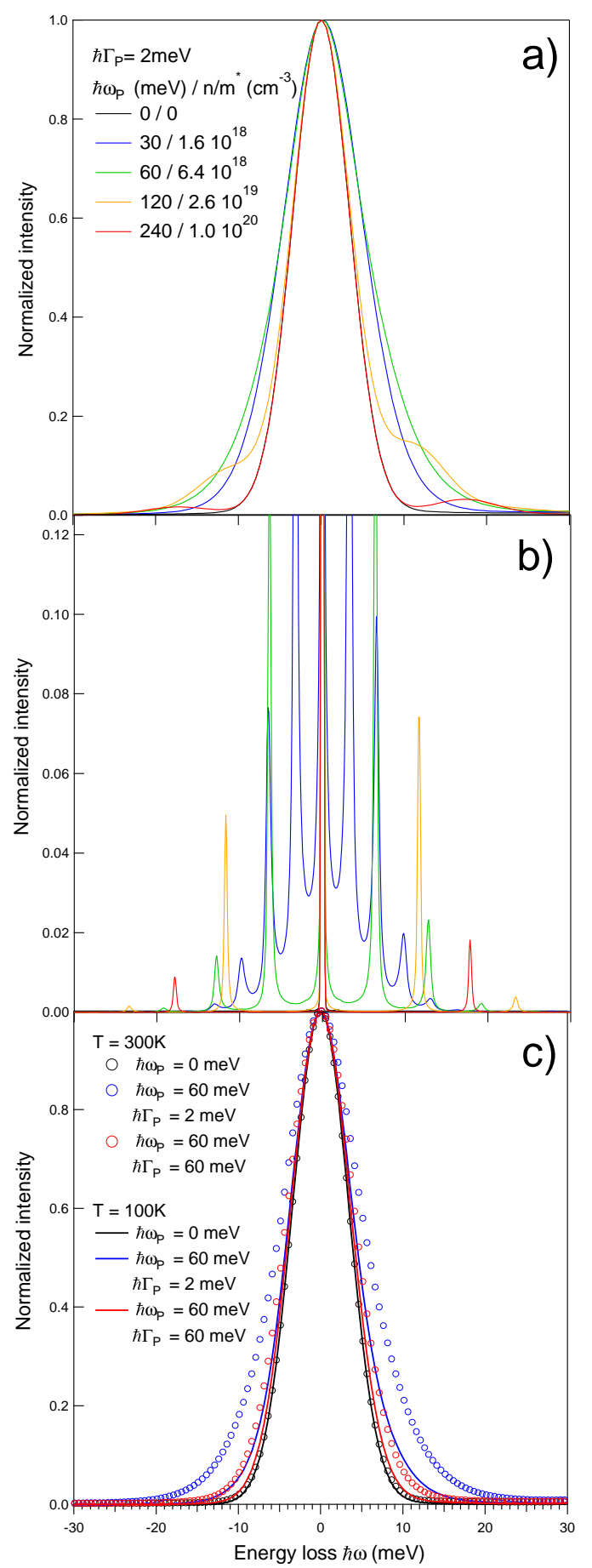

FIG. 3. Elastic peak shape a) for selected plasmon frequencies $\omega_{P}$ (or carrier density $n / m^{*}$ ) at fixed damping $\hbar \Gamma_{P}=2 \mathrm{meV}$ and room temperature, b) at reduced damping $\hbar \Gamma_{P}=0.25 \mathrm{meV}$ and increase apparatus resolution $\Delta_{a}=0.25 \mathrm{meV}(T=300 \mathrm{~K})$ and, c $)$ at two different temperatures $T=100,300 \mathrm{~K}$ for plasma frequencies $\omega_{P}, \Gamma_{P}$ given in figure. 


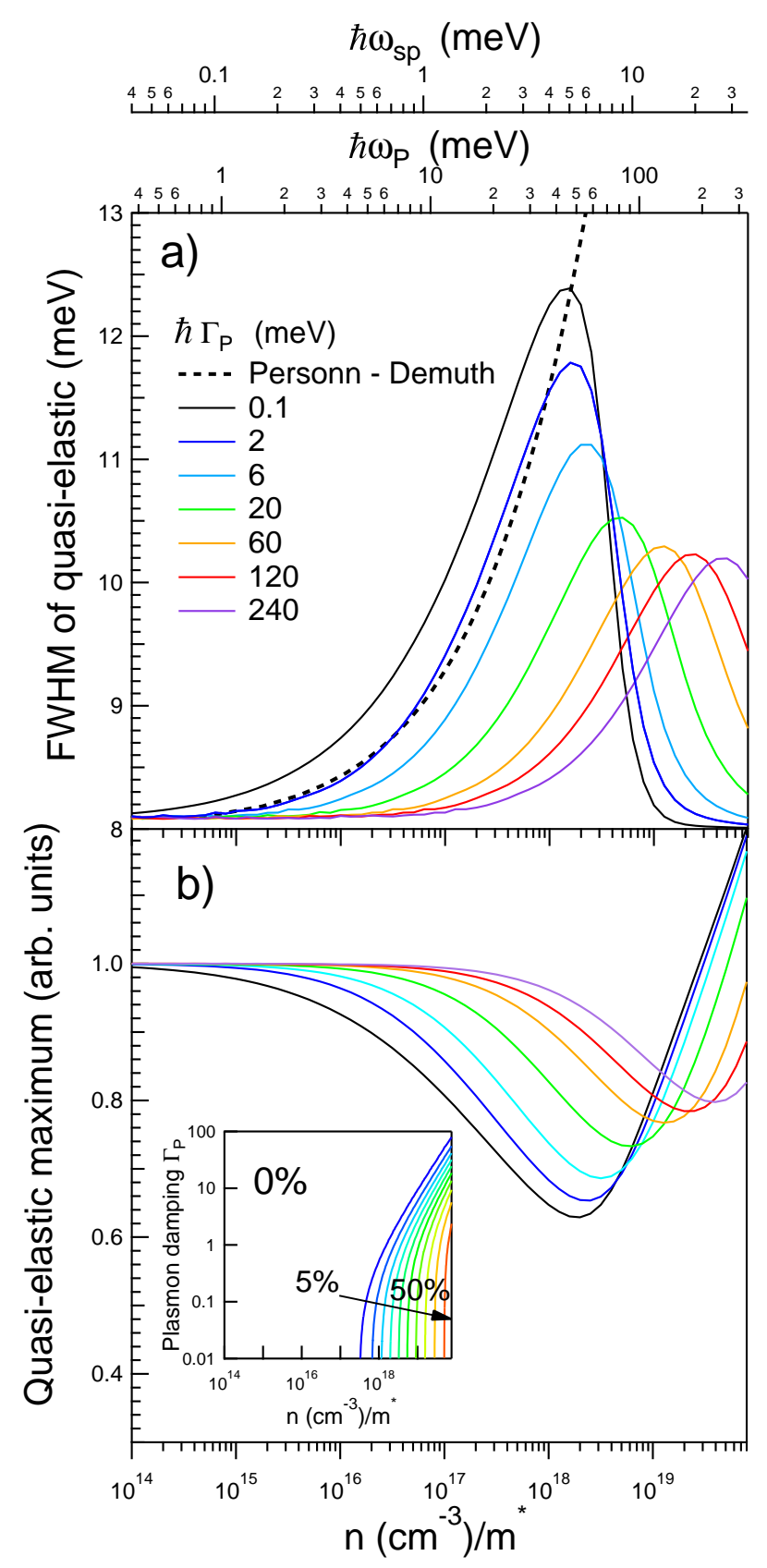

FIG. 4. a) FWHM and b) maximum of the quasi-elastic peak at $T=300 \mathrm{~K}$ as obtained from a simulated spectrum including multiple excitations (Eqs. 1-6). Quantities are plotted as a function of (i) carrier density $n$ normalized by the effective mass $m^{*}$ (bottom scale) or (ii) the bulk plasmon $\omega_{P}$ or surface plasmon $\omega_{s p}$ frequency (top scales) for various carrier plasmon dampings $\Gamma_{P}$. The comparison with the analytic formula Eq. S3 of Supplemental Material ${ }^{71}$ (dotted black line) is added for completeness in Fig. a. The inset of Fig. b shows the fraction of elastically scattered electrons versus carrier density and damping. ing the carrier concentration result in a broadening of the quasi-elastic peak up to a turn over point at which a high enough surface plasmon frequency $\omega_{s p}$ forms an isolated single excitation (Fig. 3-a,b) that appears as a side shoulder of the elastic peak. In parallel, the quasi-elastic maximum follows the reverse trend. It decreases before the turn over point and then increases up to a value higher than that of a carrier-free substrate (Fig. 3-b) which translates the increase of the elastic contribution to the quasi-elastic peak. Indeed, the progressive metallization of the substrate increases the fraction of elastically scattered electrons (inset of Fig. 4) given theoretically ${ }^{35}$ by $e^{\mathcal{P}(t=0)}$ (Eq. 3$)$.

As shown in Fig. 4 when using a typical experimental resolution of $\Delta_{a}=8 \mathrm{meV}$, increasing $\Gamma_{P}$ at fixed carrier density $n / m^{*}$ surprisingly sharpens the quasi-elastic peak down to the resolution function. Notably, the discrepancies between the present simulations and the analytic formula of the broadening of quasi-elastic peak of Ref. 43 (see Sect. S2 of Supplemental Material ${ }^{71}$ and dotted black lines in Fig. 4-a and Fig. 5) demonstrate its inadequacy in the present case due to the failure of the assumption of infinitesimal $\Gamma_{P}$ and $\omega_{s p}$.

Finally, a temperature dependence of the elastic peak is found, in line with experimental findings ${ }^{69}$, which results from a complex interplay between damping and carrier density (Fig. 5). Indeed, despite the existence of possible combinations of phonon modes at $\hbar \omega_{s p h, 2}-$ $\hbar \omega_{s p h, 1}=9.2 \mathrm{meV}$, the elastic peak without carrier contribution $\left(\omega_{P}=0\right)$ should be insensitive to temperature (Fig. 3, black curve and circles).

\section{B. Screening of phonons by carrier excitations}

The plasmon-phonon coupling was addressed by Baumard and Gervais ${ }^{66,67}$ in their infrared study of samples with variable reduction states. As shown in Figs. 6,7,8, free carriers have a triple effect on phonon losses: (i) a broadening, (ii) a variation of relative intensities and (iii) a shift toward higher frequency .

The broadening and change in intensity result from the existence of complex multiple and combination modes between surface phonon and plasmon excitations as demonstrated by the comparison of line profiles with (Fig. 6-b, Eq. 3) and without (Fig. 6-c, Eq. 3; $n=1$ ) multiple excitations. As seen in simulations at increased resolution $\left(\Delta_{a} \rightarrow 0\right)$ and reduced dampings of both phonons and plasmon $^{109}\left(\Gamma_{L O}, \Gamma_{T O}, \Gamma_{P} \rightarrow 0\right)$, the single-loss spectrum (Fig. 6-c) involves only the three main surface phonons $\omega_{s p h, 1}, \omega_{s p h, 2}, \omega_{s p h, 3}$ (plus minor modes, Tab. S1 of Supplemental Material ${ }^{71}$ ) and a surface plasmon $\omega_{s p}$. Upon including multiple excitations, the surface phonon $\omega_{s p h, 3}$ is accompanied by side shoulders at $\omega_{s p h, 3} \pm \omega_{s p}$. The resulting complex overall broadening follows roughly the 

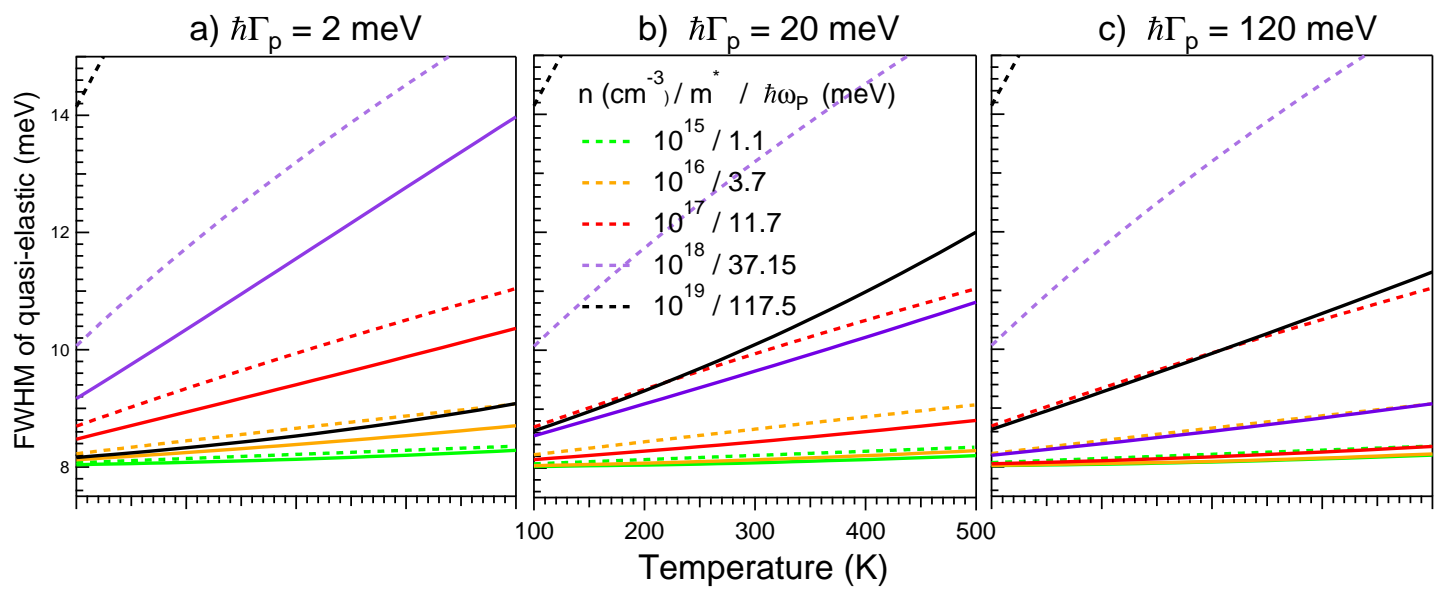

FIG. 5. Same as Fig. 4 (full lines) but as a function of temperature for various carrier densities $n / m^{*}$ (or plasmon frequencies $\left.\omega_{P}\right)$ and three different dampings: a) $\hbar \Gamma_{P}=2 \mathrm{meV}$, b) $\hbar \Gamma_{P}=20 \mathrm{meV}$, c) $\hbar \Gamma_{P}=120 \mathrm{meV}$. Data are compared to the analytic results of Eq. S3 of Supplemental Material ${ }^{71}$ (dotted lines).

trend of the quasi-elastic peak (Fig. 3-a). Indeed, if $\mathcal{P}(t)=\mathcal{P}_{P l}(t)+\mathcal{P}_{P h}(t)$ can be decomposed as the sum of isolated plasmon and phonon contributions (Eqs. 5-4), then the full spectrum

$$
\begin{aligned}
P(\omega) & =e^{-\mathcal{P}(0)} R(\omega) \otimes\left\{\delta(\omega)+\sum_{n=1}^{\infty} \frac{1}{n !}\left[\mathcal{P}_{P l}(\omega)\right]^{n \otimes}\right\} \\
& \otimes\left\{\delta(\omega)+\sum_{n=1}^{\infty} \frac{1}{n !}\left[\mathcal{P}_{P h}(\omega)\right]^{n \otimes}\right\}
\end{aligned}
$$

appear as the convolution of the phonon multiple excitations by the plasmonic ones and vice-versa. However, depending on the overlap in $\mathcal{P}(t)$, both excitations can even mix to generate the so-called "plasmaron" $39,40,110$.

Regarding shifts, low energy phonons at $\hbar \omega_{s p h, 1} \simeq 45.2 \mathrm{meV}$ and $\hbar \omega_{s p h, 2} \simeq 54.4 \mathrm{meV}$ are much less affected than the peak $\hbar \omega_{s p h, 3} \simeq 92.9 \mathrm{meV}$ which is the focus of Fig. 7. This is particularly clear in Fig. 8 where fictitious spectra are plotted in the single loss approximation at reduced damping; the shift, which directly stems from the screening of phonon excitation by carriers, correlates with the appearance of the surface plasmon feature close to the elastic peak. Up to a carrier density of $n / m^{*} \simeq 510^{17} \mathrm{~cm}^{-3}$ $\left(\hbar \omega_{P} \simeq 20 \mathrm{meV}\right)\left(\right.$ Fig. 7-8), $\hbar \omega_{s p h, 3}$ keeps its unscreened value of $95.4 \mathrm{meV}$ with a slight decrease in intensity due to broadening. Phonon intensity and $\Gamma_{P}$-induced broadening are strongly correlated (Fig. 7-b); the higher the plasmon damping $\Gamma_{P}$ the lower the intensity as expected from the presence of $\omega_{s p h, 3} \pm \omega_{s p}$ peaks. Above $n / m^{*} \simeq 510^{17} \mathrm{~cm}^{-3}$, a noticeable shift is observed.

To first order, these findings can be rationalized in the single excitation regime by accounting for the high energy phonon with a model of isolated oscillator of strength
$\Omega$, frequency $\omega_{0}$ and damping $\Gamma$ screened by a constant background $\epsilon_{c}$ :

$$
\epsilon_{o s c}(\omega)=\epsilon_{c}+\frac{\Omega^{2}}{\omega_{0}^{2}-\omega^{2}-i \Gamma \omega} .
$$

Close to $\omega_{0}$, the corresponding loss function:

$$
\operatorname{Im}\left[\frac{1}{1+\xi_{o s c}(\omega)}\right]=\frac{\Gamma \omega \Omega^{2}}{\left[\left(1+\epsilon_{c}\right)\left(\omega_{0}^{2}-\omega^{2}\right)+\Omega^{2}\right]^{2}+\Gamma^{2} \omega^{2}\left(1+\epsilon_{c}\right)^{2}}
$$

displays a quasi-lorentzian shape ${ }^{35}$ centered on $\omega_{o s c}$, of intensity $I_{o s c}$ and of FWHM $W_{o s c}$ given by:

$$
\begin{aligned}
\omega_{o s c} & =\omega_{0}+\frac{\Omega^{2}}{2 \omega_{0}\left(1+\epsilon_{c}\right)}, \\
I_{o s c} & =\frac{\Omega^{2}}{\Gamma \omega_{0}\left(1+\epsilon_{c}\right)^{2}}, \\
W_{o s c} & =\frac{\Omega^{2}}{\omega_{0}\left(1+\epsilon_{c}\right)}-\Gamma .
\end{aligned}
$$

In the case of plasmon (see Sect. III D), the screening constant at the oscillator frequency $\epsilon_{c} \simeq \epsilon_{c, 0}-\omega_{P}^{2} / \omega_{0}^{2}$ decreases with the plasmon frequency below the value given by the remaining excitations $\epsilon_{c, 0}$. For plasmon frequencies smaller than the oscillator eigen-frequency $\omega_{0}$ or more precisely when $\omega_{P} \ll \omega_{0} \sqrt{1+\epsilon_{c, 0}}$, a first order expansion of Eqs. 17-19 shows that the frequency shift, the variation of intensity and the width vary linearly with the carrier density $n \sim \omega_{P}^{2}$ with slopes $\alpha_{P}$ and $\beta_{P}$ given by:

$$
\begin{gathered}
\Delta \omega_{o s c}=\alpha_{P} \omega_{P}^{2}=\frac{\Omega^{2} \omega_{P}^{2}}{2 \omega_{0}^{3}\left(1+\epsilon_{c, 0}\right)^{2}}, \\
\Delta I_{o s c} / I_{o s c}=\beta_{P} \omega_{P}^{2}=\frac{2 \omega_{P}^{2}}{\omega_{0}^{2}\left(1+\epsilon_{c, 0}\right)^{2}}, \\
\Delta W_{o s c}=2 \alpha_{P} \omega_{P}^{2}=\frac{\Omega^{2} \omega_{P}^{2}}{\omega_{0}^{3}\left(1+\epsilon_{c, 0}\right)^{2}} .
\end{gathered}
$$




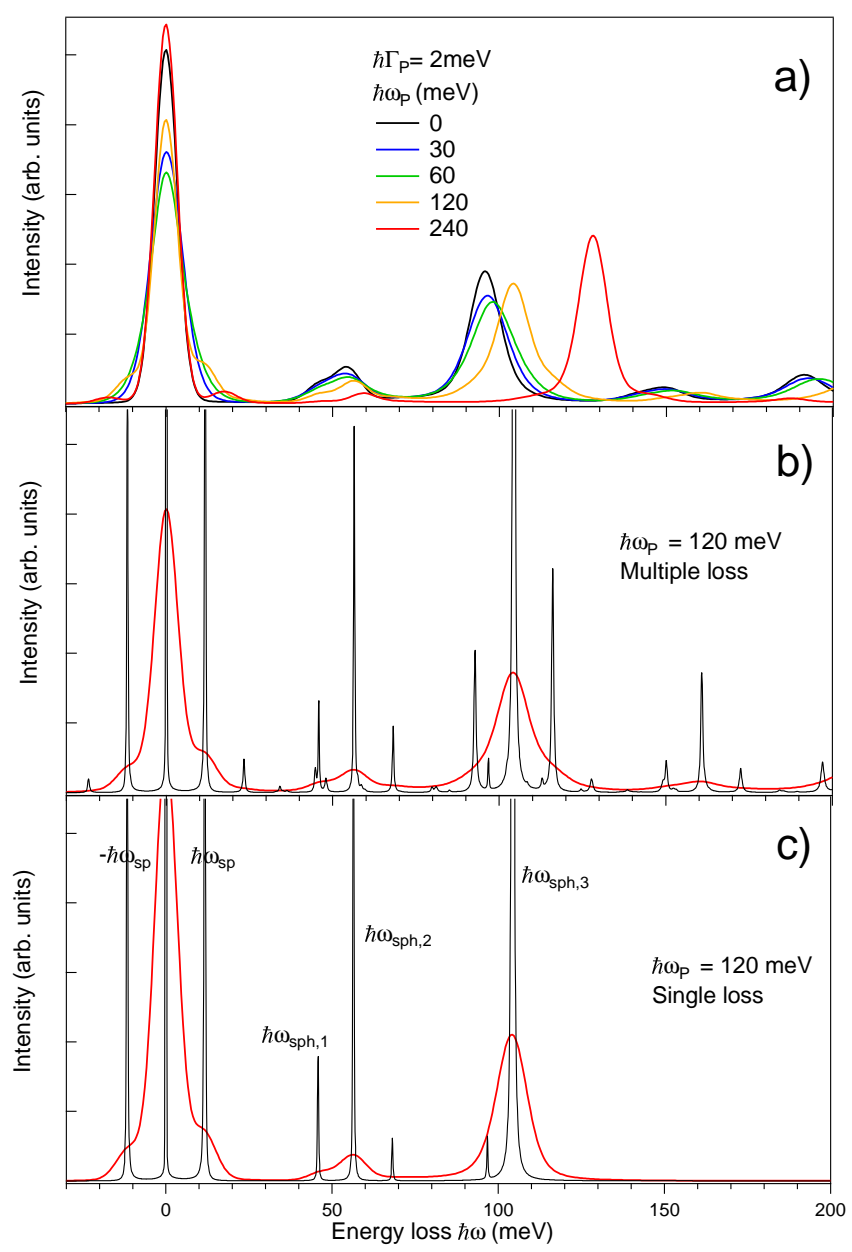

FIG. 6. a) Evolution of the phonon energy loss region with the plasmon frequency $\omega_{P}$ at fixed damping $\hbar \Gamma_{P}=2 \mathrm{meV}$. b) Multiple and c) single losses (red line) for the $\hbar \omega_{P}=120 \mathrm{meV}$ case are compared to simulations at reduced phonon $\hbar \Gamma_{T O}=$ $\hbar \Gamma_{L O}=0.25 \mathrm{meV}$ and plasmon $\hbar \Gamma_{P}=0.25 \mathrm{meV}$ dampings and increased resolution $\Delta_{a}=0.25 \mathrm{meV}$ (black lines).

The estimated values of the corresponding parameters are given in Tab. S2 of Supplemental Material ${ }^{71}$. The linearity of the phonon shift with $\omega_{P}^{2}$ is correctly verified below $\left(\omega_{P}^{m}\right)^{2}$ on full simulations (see insets of Fig. 7 for $\left.\omega_{s p h, 3}\right)$. However, the model remains qualitative. By using the oscillator strengths of Ref. 79 and $\epsilon_{c, 0}$ obtained from the parametrization of Ref. 10, the values $\alpha_{P}$ (Tab. S2 of Supplemental Material ${ }^{71}$ ) of the slopes including all multiple excitations are systematically larger than those obtained through the screened single excitation of an isolated phonon. The discrepancy comes from the simplifying assumption of independent oscillators, since a shift of a given peak significantly change the screening of its neighbors; due to a larger oscillator strength, $\omega_{s p h, 3}$ is expected to shift faster than $\omega_{s p h, 2}$ which decreases its screening $\epsilon_{c, 0}$ and therefore increases its $\alpha_{P}$. The same reasoning applies to $\omega_{s p h, 1}$ and its

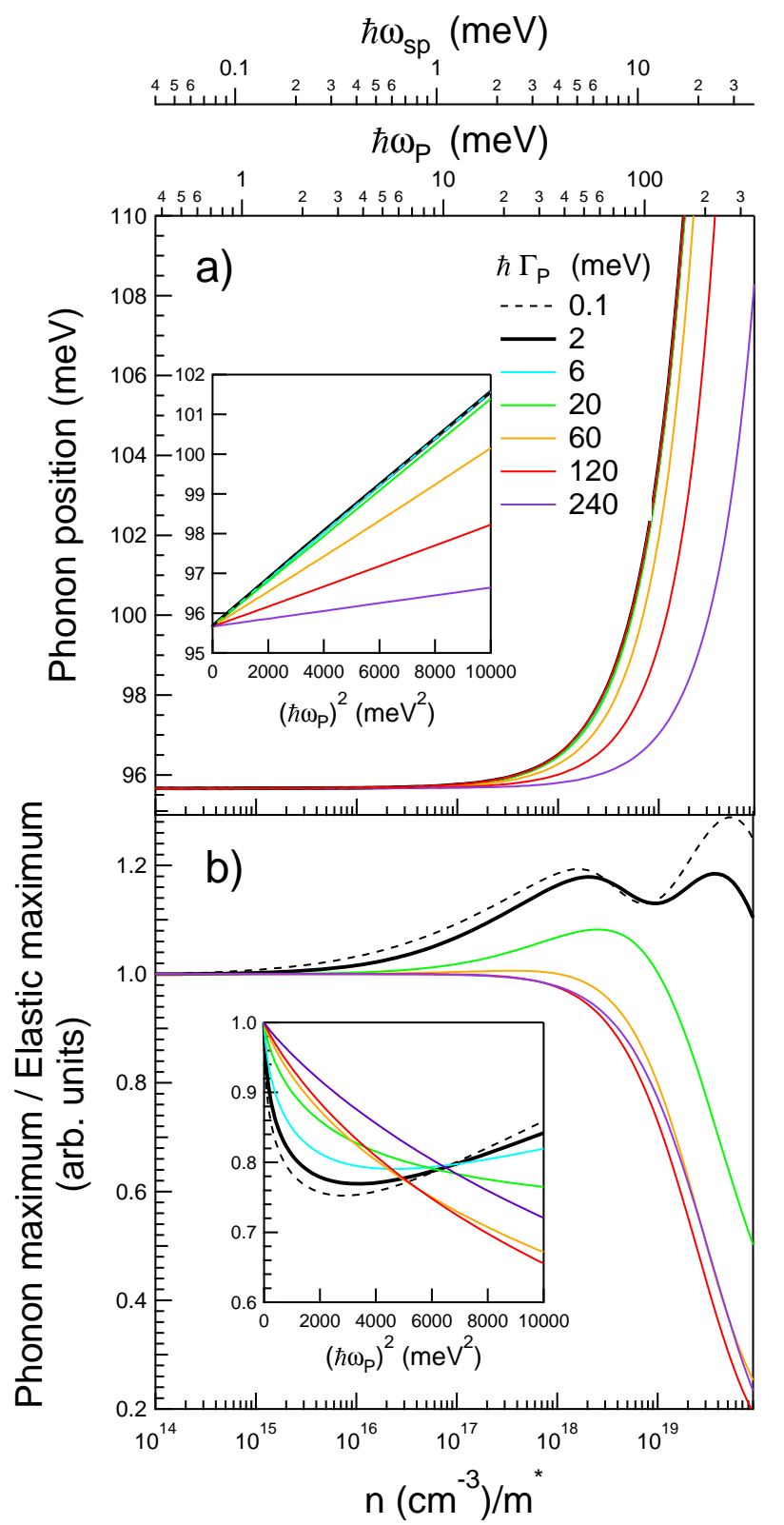

FIG. 7. Dependence on carrier density $n / m^{*}$ (bottom scale) or plasmon frequencies $\omega_{P}, \omega_{s p}$ (top scales) for various dampings $\Gamma_{P}$ of the $\omega_{s p h, 3}$-phonon: a) peak position and b) peak intensity normalized to elastic intensity. Simulations include multiple excitations. The insets show the phonon position and its intensity as a function of $\omega_{P}^{2}=n e^{2} / m_{e} m^{*} \epsilon_{0}$ once normalized to elastic.

neighbors at high energy $\omega_{s p h, 2}$. Anyway, according to the insets of Fig. 7, if all multiple excitations are taken into account, $\omega_{s p h, 3}$ is expected to shift by several $\mathrm{meV}$, while increasing $\omega_{P}$ up to $100 \mathrm{meV}$ and therefore the carrier concentration up to $n / m^{*}=7.010^{18} \mathrm{~cm}^{-3}$. 


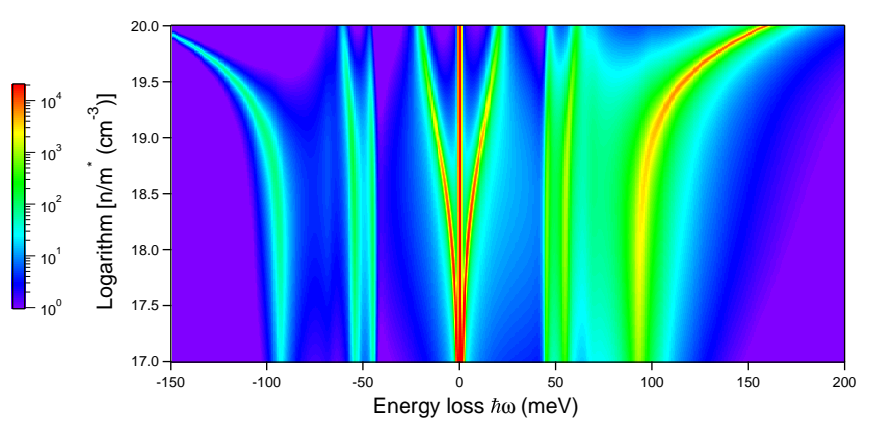

FIG. 8. Map of HREELS spectra in the single loss approximation at enhanced resolution $\Delta_{a}=1 \mathrm{meV}$ and poor phonon/plasmon damping $\hbar \Gamma=1 \mathrm{meV}$ as a function of carrier density $n / m^{*}$. Notice the correlation between the appearance of the plasmon feature and the strong shift of the high energy surface phonon $\omega_{s p h, 3}$.

\section{Screening of phonons by band gap states}

The impact of BGS on polar phonons of $\mathrm{TiO}_{2}$ was stressed in the earlier HREELS approaches ${ }^{19,20,59}$ and in an indirect way in infrared study ${ }^{66,67}$, as modification of the dielectric function at infinity $\epsilon_{\infty}$ for phonons (see Eq. 8, Sect. III A).

EELS spectra with a beam energy $E_{I}=38 \mathrm{eV}$ and a resolution $\Delta_{a}=50 \mathrm{meV}$ similar to experiments ${ }^{22,69}$ have been calculated. As a first step, the BGS is represented by an oscillator (Eq. 11, Sect. III C) of strength $\Omega_{g s}$ spread homogeneously in a semi-infinite substrate (Fig. 9-a inset). It appears as a low intensity feature between the phonon clump and the band-to-band transitions located above the $3.2 \mathrm{eV}$ band gap of rutile. Besides an obvious increase of its intensity with $\Omega_{g s}$, the BGS shifts to higher energy (Fig. 11-a) and gets asymmetric because of multiple excitations; in particular, a second order excitation appears in the inset of Fig. 9-a at around $2500 \mathrm{meV}$ for $\hbar \Omega_{g s}=2000 \mathrm{meV}$. As shown by Eq. 17 for the simple oscillator used here to describe BGS, this shift roots into the EELS cross section which is proportional to $\operatorname{Im}[1 / 1+\epsilon(\omega)]$ and is linear in $\Omega_{g s}^{2} \bmod -$ ulo the sensitivity function as shown in Fig. 11-a.

In contrast to interband transitions which seem insensitive to BGS because of their much smaller oscillator strengths, the phonon region is strongly screened by BGS. The effect is fairly well illustrated in high resolution spectrum including (Fig. 9-a) or not (Fig. 10) multiple losses, but it is less obvious at moderate resolution as usually used in EELS (Fig. 9-a, inset). Phonons evolve in the opposite direction with respect to the screening induced by carrier excitations; they all redshift and the $\omega_{s p h, 3}$ intensity decreases (Fig. 11-b). Phonon screening by the tail of BGS excitation is again the main driving force. But, since the frequency of BGS is larger than those of
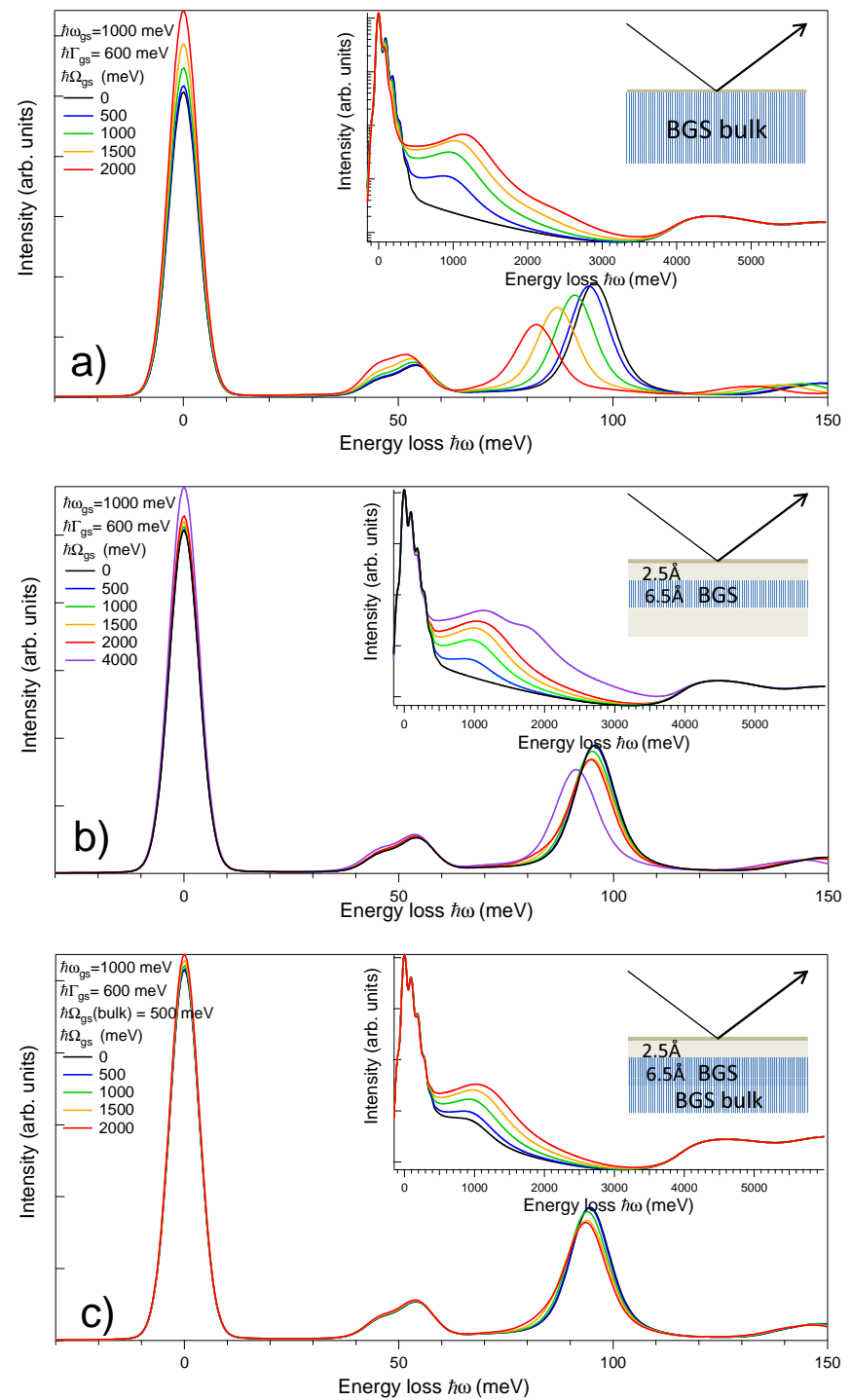

FIG. 9. Evolution of the phonon (main graphs, linear scale) and band gap (insets, logarithmic scale) electron energy loss spectra as a function of the band gap state strength $\Omega_{g s}$ (at constant frequency $\hbar \omega_{g s}=1000 \mathrm{meV}$ and damping $\hbar \Gamma_{g s}=$ $600 \mathrm{meV}$ ): a) semi-infinite substrate, b) BGS localized in a layer thickness $6.5 \AA$ just below the surface, and c) mixture of both configurations with $\hbar \omega_{g s}^{b u l k}=500 \mathrm{meV}$. Dielectric profile is chosen accordingly to the experimental findings ${ }^{22,69}$.

phonons, the real part of the BGS dielectric component $\epsilon_{g s}(\omega)$ is negative at phonon frequencies (Fig. 1) at the opposite to the plasmon contribution $\epsilon_{p l}(\omega)$. The effect can also be accounted by describing phonons through isolated oscillators to determine variations in position and intensity. To first order i.e. $\quad \Omega_{g s} \ll \omega_{g s}^{m}=\omega_{g s} \sqrt{1+\epsilon_{c, 0}}$, they are linear in $\Omega_{g s}^{2}$ with negative slopes that amount 


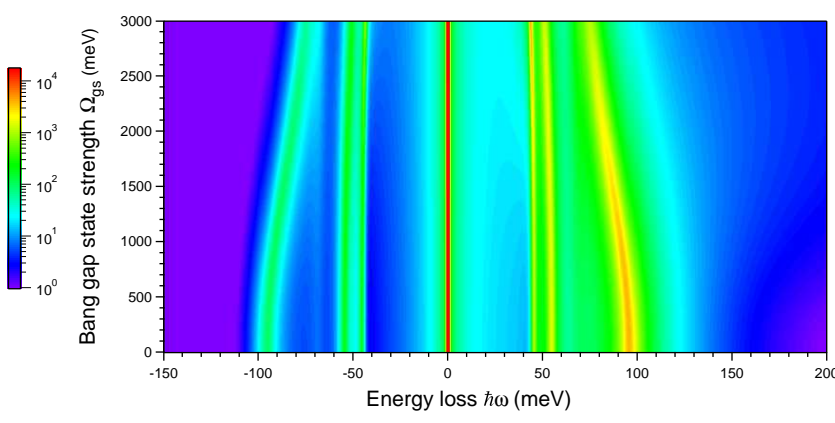

FIG. 10. Map of HREELS spectra of phonons in the single loss approximation at enhanced resolution $\Delta_{a}=1 \mathrm{meV}$ and poor phonon/plasmon damping $\hbar \Gamma=1 \mathrm{meV}$ as a function of band gap state strength $\Omega_{g s}$. A clear downward shift of the surface phonons, in particular $\omega_{s p h n, 3}$ is observed.

to:

$$
\begin{gathered}
\Delta \omega_{o s c}=-\alpha_{g s} \Omega_{g s}^{2}=-\frac{\Omega^{2} \Omega_{g s}^{2}}{2 \omega_{0} \omega_{g s}\left(1+\epsilon_{c, g s}\right)^{2}}, \\
\Delta I_{o s c} / I_{o s c}=-\beta_{g s} \Omega_{g s}^{2}=-\frac{2 \Omega_{g s}^{2}}{\omega_{g s}^{2}\left(1+\epsilon_{c, g s}\right)^{2}}, \\
\Delta W_{o s c}=-2 \alpha_{g s} \Omega_{g s}^{2}=-\frac{\Omega^{2} \Omega_{g s}^{2}}{\omega_{0} \omega_{g s}\left(1+\epsilon_{c, g s}\right)^{2}} .
\end{gathered}
$$

Downward shifts and decrease in phonon intensities obtained in the case of multiple excitations (Fig. 11-a) are well accounted for. The limits of the modelling by isolated oscillators and single loss explain the quantitative discrepancy between calculated slopes $\alpha_{g s}, \beta_{g s}$ and actual values (see Tab. S2 of Supplemental Material ${ }^{71}$ for numerical values).

Surprisingly, the BGS also impacts the quasi-elastic peak intensity which increases at constant FWHM (Fig. 11-a) with $\Omega_{g s}$. This phenomenon finds its origin in the transfer of intensity from phonons and their multiple excitations to the elastic contribution of the quasielastic peak. Therefore, once normalized to the elastic peak, phonons decrease in intensity upon increasing the BGS strength (Fig. 11-b inset). This relative variation of phonon/elastic appears in an obvious way on experimental spectra during healing of defects by oxygen exposure $^{69}$.

\section{THE EELS DEPTH SENSITIVITY AND THE PROFILE OF DIELECTRIC FUNCTION}

Up to now, the discussion was restricted to a semiinfinite substrate. The profile of dielectric function related to defects was not accounted for. However, while both surface oxygen vacancies and titanium interstitials contribute to BGS in $\mathrm{TiO}_{2}{ }^{22}$, excess electrons were shown to be localized on subsurface titanium by resonant photoemission ${ }^{16}$ and futher confirmed by out-of-specular EELS $^{22}$. In this context, the variable depth sensitivity of EELS in dipolar regime as a function of impact and loss energies is of interest. A fair estimate of probing $\operatorname{depth}^{35,50}$ is the evanescent decay length $d_{p}$ of the electric field due to the incoming electron ${ }^{35}$ which is given by the inverse of the electron wavevector transfer $d_{p} \simeq 1 / k_{\|}=1 /\left(k_{S}-k_{I}\right) \sin \Theta_{I} \simeq 1 /\left(k_{I} \theta_{E} \sin \Theta_{I}\right) . \quad d_{p}$ has been plotted in Fig. 12 for two beam energies $E_{I}=8$ and $38 \mathrm{eV}$, typical for loss regimes of phonons and BGS and compared to values averaged over the detector aperture $^{22}$. The higher the beam energy and the lower the energy loss the deeper the probing depth. Measurements are bulk sensitive for phonons $\left(d_{p} \simeq 200 \AA\right)$, not speaking about quasi-elastic excitations. Conversely, EELS at $E_{I}=38 \mathrm{eV}$ that probes the subsurface at the BGS energy $\left(d_{p} \simeq 30 \AA\right)$ becomes very surface sensitive for interband transition $\left(d_{p}<10 \AA\right)$.

But, caution is required for detailed analysis since the depth dependence of EELS cross section is quite complex. For instance, while it corresponds to a constant dielectric function, the sensitivity function $F\left(E_{I}, \cos \Theta_{I}, \omega\right)$ that takes into account the scattering geometry $^{35}$ (Eq. 1) does not follow the $d_{p}(\omega)$ trend (Fig. 12; symbols vs lines). A better definition of $d_{p}$ must account for the weight given for each $k_{\|}$by the sensitivity function ${ }^{50}$ and for the $k_{\|}$-dependence of the loss function $G\left(k_{\|}, \omega\right)$ (Eq. 1) which expression is already complex for a stratified medium ${ }^{58}$ (Eq. S2 of Supplemental Material ${ }^{71}$ ). Therefore, it is mandatory to resort to full numerical simulations to accurately discuss depth sensitivity effects and profile of dielectric function, as shown in the following three examples.

In Fig. 9, several models of distribution of BGS (bulk: Fig. 9-a, subsurface: Fig. 9-b, and mixture of both: Fig. 9-c) have been compared at increasing BGS oscillator strength. As shown in the schematic profiles in insets, subsurface BGS are put in a $6.5 \AA$ thick layer below a $2.5 \AA$ dead-layer to mimic the localization of charge on subsurface Ti atoms of the rutile $\mathrm{TiO}_{2}(110)^{16,22}$. The sizable impact of bulk BGS on phonon position and on relative intensity to elastic peak (Fig. 11-a), is considerably damped for a subsurface excitation despite a similar intensity as a band gap feature in EELS (Fig. 9-b). This effect finds its origin in the difference of probing depth between excitations at phonon and band gap state energies (Fig. 12). In a similar way, the barely visible bulk BGS excitation buried at a depth below $6.5+2.5=9 \AA$ (Fig. 11-c; black line in inset) strongly shifts the main phonon peak (Fig. 11-b and Fig. 11-c; black lines).

Fig. 13 illustrates the more complex dielectric function profile of an exponential decaying gradient of carriers from a surface value $\hbar \omega_{P}=120 \mathrm{meV}$ to a bulk value $\hbar \omega_{P}=0 \mathrm{meV}$, which could mimic an accumulation 

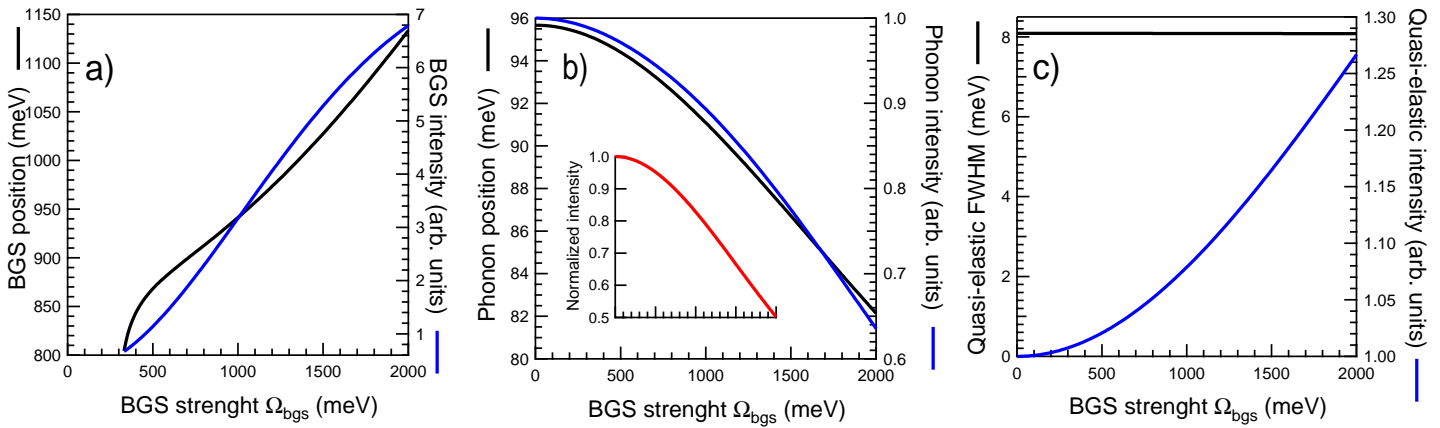

FIG. 11. Variation with the band gap state strength $\Omega_{g s}$ of the peak intensities, positions or FWHMs of the a) BGS, b) phonon and c) elastic peak. Notice the BGS is a maximum-less shoulder below a critical $\Omega_{g s}$ value. The phonon intensity normalized to the elastic intensity is shown in inset of Fig.-b. All multiple losses have been accounted for.

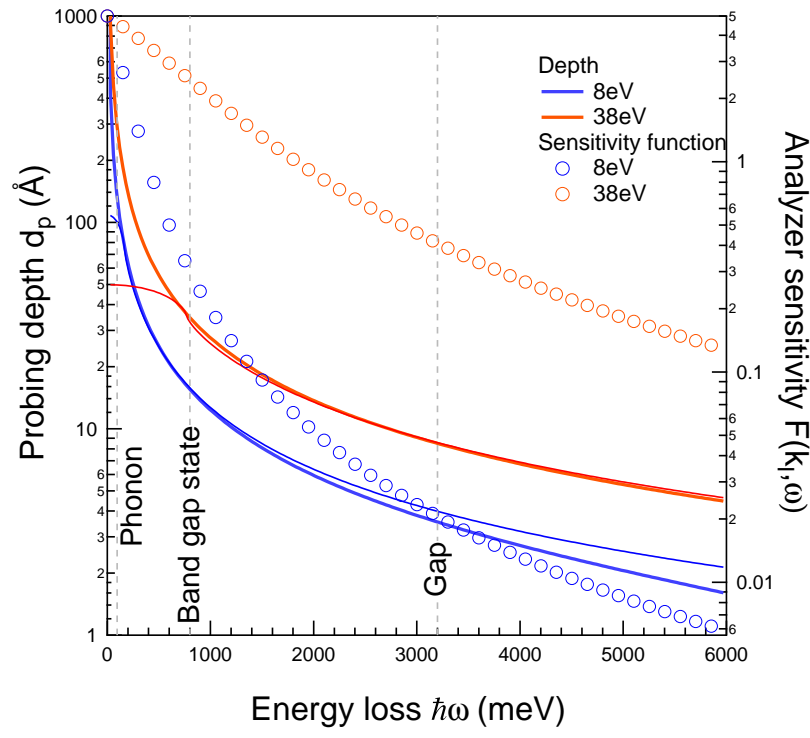

FIG. 12. Probing depths (left scale) and analyser sensitivity function (right scale) for two beam energies $E_{I}=8 \mathrm{eV}$ and $38 \mathrm{eV}$. The basic estimate $d_{p}$ (thick lines) is compared to the average $\left\langle d_{p}\right\rangle$ over a circular slit of aperture $\theta_{c}=1^{\circ}$ (thin lines). The main effect of slit integration is to smoothen the divergence at $\hbar \omega=0^{22}$. Phonon, band gap state and band gap of $\mathrm{TiO}_{2}$ are shown by dotted lines. Notice that $d_{p}$ was also calculated well outside the regime of negligible loss at $E_{I}=8 \mathrm{eV}$.

layer due to band-bending and charge transfer with surface defects. The profile was sampled in a stair case way (inset of Fig. 13) over 3 times the decay length $\Lambda$ and the loss function computed recursively (Eq. S2 of Supplemental Material $\left.{ }^{71}\right)$. The effects on the quasielastic peak, the phonon position and their relative ratio parallels the trends described in Sects. IV B-IV C for a semi-infinite sample. Interestingly, the spectrum poorly evolves between $\Lambda=2000 \AA$ (violet curve) and a bulk substrate (bold black line) in the phonon range, while

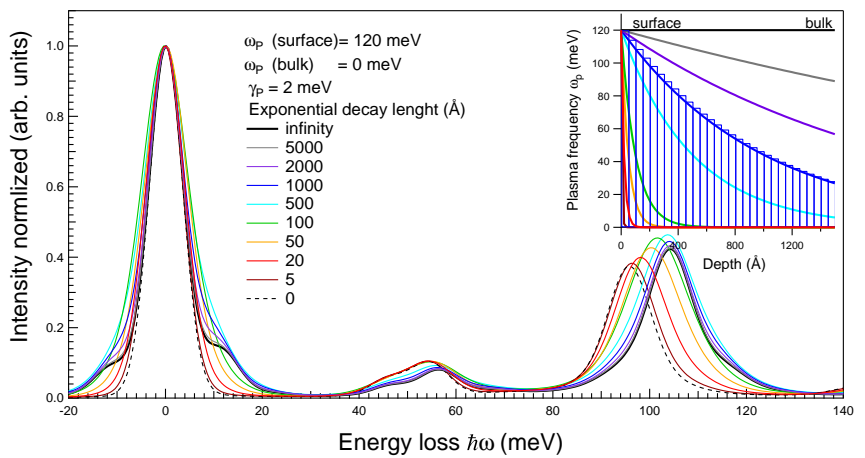

FIG. 13. Effect of an exponential gradient of carrier density (shown in inset) on the HREELS phonon spectrum in the absence of BGS contribution. The plasma frequency goes from $\hbar \omega_{P}=120 \mathrm{meV}$ at the surface to $\hbar \omega_{P}=0 \mathrm{meV}$ in the bulk while the damping is taken constant equal to $\hbar \Gamma_{P}=2 \mathrm{meV}$. The exponential decay length goes from $0 \AA$ to infinity i.e. from an undoped substrate to flat profile. The profile is sampled over 60 slices in between 0 and $3 \Lambda$ as illustrated by the blue staircase curve for $\Lambda=1000 \AA$. Spectra are normalized to the elastic peak maximum.

sizeable differences are still visible for the quasi-elastic peak. The explanation lies in the difference of probing depth (see Fig. 12) at the two energies. The sensitivity to such high $\Lambda$-values for phonons compared to the expected probing depth $d_{p} \simeq 200 \AA$ comes from the initial difference of slopes of $\omega_{P}(z)$; this means that a linear profile would give a quite similar spectrum.

The last example (Fig. 14) deals with a fictitious highly conductive layer $\left(\hbar \Gamma_{p}=2 \mathrm{meV}\right)$ of thickness $(t=6.5 \AA)$ equivalent to the distance between Ti planes in $\mathrm{TiO}_{2}(110)$ buried at increasing depth below the surface. Its plasma frequency $\hbar \omega_{P}=500 \mathrm{meV}$ corresponds to a surface carrier density of $n_{S} / m^{*}=1.210^{13} \mathrm{~cm}^{2}$ typical for a doping by a fraction of monolayer of 


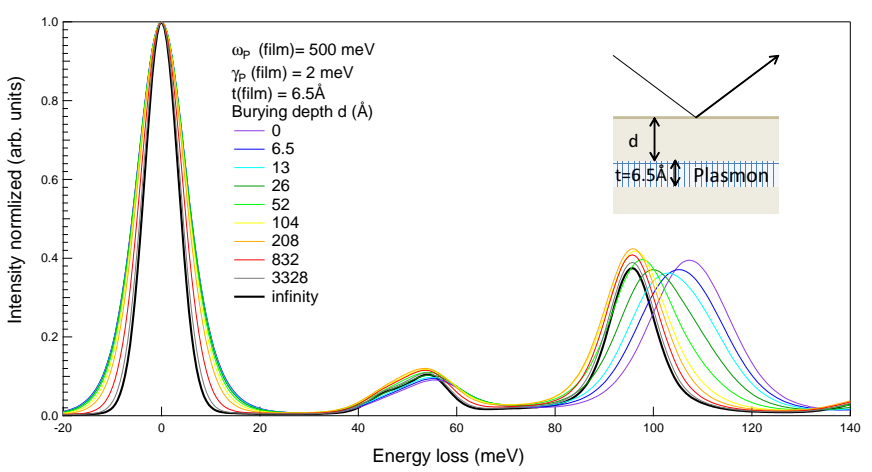

FIG. 14. Effect of a conductive layer $\left(\hbar \omega_{P}=500 \mathrm{meV}, \hbar \Gamma_{p}=\right.$ $2 \mathrm{meV}, t=6.5 \AA$ ) buried at different depth $d$ on the HREELS phonon spectrum (see inset). Spectra are normalized to the elastic peak maximum.

defects. This layer impacts the phonon position $\omega_{s p h, 3}$ down to around the expected probing depth of $1 / k_{\|}=d_{p} \simeq 200 \AA$, but the peak intensity is not yet converged. The surface plasmon excitation confined in this layer are still able to broaden the quasi-elastic peak up to a rather large burying depth.

These few theoretical examples highlight the sensitivity of EELS to profiles of dielectric function and the complexity of interplay between excitations.

\section{CONCLUSION}

Based on an accurate build-up of the dielectric function from far-infrared to ultraviolet, the interplay between the solid-state excitations of reduced $\mathrm{TiO}_{2}$ rutile was explored in the frame of the EELS dipole scattering theory. While the contributions from optical phonons and interband transitions were extracted from literature, the transport of excess electrons was described through a Drude model and the associated band gap states through an oscillator. The analysis showed that the dielectric anisotropy has a minor impact due to a fortuitous degeneracy of phonon modes. Upon increasing the carrier concentration or the plasma frequency and contrary to most semi-conductors, only a modest broadening of the quasi-elastic peak is expected because of the large static dielectric function and effective mass in $\mathrm{TiO}_{2}$. Only in the most extreme case, a surface plasmon shoulder on the side of the quasi-elastic peak is to be detected. Nevertheless, carriers should induce a measurable temperature dependence of this latter.

Conversely, band gap states appear clearly as isolated features. Due to its large oscillator strength, the surface phonon around $95 \mathrm{meV}$ can be used as a reliable reporter of the excess electrons excitations through the screening they induce. Above a carrier concentration of $10^{17}-10^{18} \mathrm{~cm}^{-3}$, an upward shift is predicted. However, it should be counterbalanced by a downward shift due to band gap states oscillator strength. Both plasmon and BGS impact the ratio of phonon to elastic peak by pumping out intensity. If the screening effect can be rationalized through an isolated oscillator model of phonons, the accurate description of shifts, variations of intensity or broadenings of phonons and elastic peak requires a full account of all excitations. At last, while probing depth is much larger for the quasi-elastic peak and phonons than for band gap states, the previous trends obtained for semi-infinite substrate hold true for various dielectric profiles. But the account of the probing depth turns out to be mandatory for quantitative analysis.

The present conclusions on phonon screening in EELS can be generalized to any reducible oxide showing polaronic defect states since a high static dielectric constant is often related to longitudinal phonon modes of high oscillator strengths.
* Corresponding author :remi.lazzari@insp.jussieu.fr

1 J. Jupille and G. Thornton, Defects at oxide surfaces (Springer, 2015).

${ }^{2}$ U. Diebold, Surf. Sci. Rep. 48, 53 (2003).

3 C. Lun Pang, R. Lindsay, and G. Thornton, Chem. Soc. Rev. 37, 2328 (2008).

4 L.-M. Liu, P. Crawford, and P. Hu, Prog. Surf. Sci. 84, 155 (2009)

5 Z. Dohnálek, I. Lyubinetsky, and R. Rousseau, Prog. Surf. Sci. 85, 161 (2010).

6 M. A. Henderson, Surf. Sci. Rep. 66, 185 (2011).

7 C. L. Pang, R. Lindsay, and G. Thornton, Chem. Rev. 113, 3887 (2013).

8 M. A. Henderson and I. Lyubinetsky, Chem. Rev. 113, 4428 (2013).

${ }^{9}$ W.-J. Yin, B. Wen, C. Zhou, A. Selloni, and L. M. Liu,
Surf. Sci. Rep. 73, 58 (2018).

10 D. Schöche, T. Hofmann, R. Korlacki, T. Tiwald, and M. Schubert, J. Appl. Phys. 113, 164102 (2013).

11 D. Emin, Phys. Rev. B 48, 13691 (1993).

12 A. Fujimori, A. E. Bocquet, K. Morikawa, K. Kobayashi, I. T. Saithoh, Y. Tokura, I. Hasex, and M. Onoda, J. Phys. Chem. Solids 57, 1379 (1996).

13 G. Mattioli, P. Alippi, F. Filippone, R. Caminiti, and A. A. Bonapasta, J. Phys. Chem. C 114, 21694 (2010).

14 V. E. Henrich, G. Dresselhaus, and H. J. Zeiger, Phys. Rev. Lett. 36, 1335 (1976).

15 R. Kurtz, R. Stockbauer, T. Madey, E. Román, and J. de Segovia, Surf. Sci 218, 178 (1989).

16 P. Krüger, S. Bourgeois, B. Domenichini, H. Magnan, D. Chandesris, P. Le Fèvre, A. M. Flank, J. Jupille, L. Floreano, A. Cossaro, A. Verdini, and A. Morgante, 
Phys. Rev. Lett. 100, 055501 (2008).

17 C. M. Yim, C. L. Pang, and G. Thornton, Phys. Rev. Lett. 104, 036806 (2010).

18 P. Borghetti, E. Meriggio, G. Rousse, G. Cabailh, R. Lazzari, and J. Jupille, J. Phys. Chem. Lett. 7, 3223 (2016).

19 G. Rocker, J. A. Schaefer, and W. Göpel, Phys. Rev. B 30, 3704 (1984).

20 S. Eriksen and R. G. Egdell, Surf. Sci. 180, 263 (1987).

21 W. S. Epling, C. H. F. Peden, M. A. Henderson, and U. Diebold, Surf. Sci. 412-413, 333 (1998).

22 J. Li, R. Lazzari, S. Chenot, and J. Jupille, Phys. Rev. B Rapid Comm. 97, 041403(R) (2018).

23 A. C. Papageorgiou, N. S. Beglitis, C. L. Pang, G. Teobaldi, G. Cabailh, Q. Chen, A. J. Fisher, W. A. Hofer, and G. Thornton, Proc. Natl. Acad. Sci. USA 107, 2391 (2010).

24 M. Setvin, C. Franchini, X. Hao, M. Schmid, A. Janotti, M. Kaltak, G. Van de Walle, G. Kresse, and U. Diebold, Phys. Rev. Lett. 113, 086402 (2014).

25 V. Bogomolov and D. Mirlin, Phys. Stat. Sol 27, 443 (1968).

26 V. M. Khomenko, K. Langer, H. Rager, and A. Fett, Phys. Chem. Miner. 25, 338 (1998).

27 M. Chiesa, M. Paganini, S. Livraghi, and E. Giamello, Phys. Chem. Chem. Phys. 15, 9435 (2013).

28 L. Hollander and P. L. Castro, Phys. Rev. 119, 1882 (1960).

29 J. H. Becker and W. R. Hosler, Phys. Rev. 137, A1872 (1965).

30 E. Iguchi, K. Yajima, T. Asahina, and Y. Kanamori, J. Phys. Chem. Solids 35, 597 (1974).

31 E. Yagi, R. R. Hasiguti, and M. Aono, Phys. Rev. B 54, 7945 (1996).

32 E. Hendry, F. Wang, J. Shan, T. F. Heinz, and M. Bonn, Phys. Rev. B 69, 081101(R) (2004).

33 P. M. Kowalski, M. F. Camellone, N. N. Nair, B. Meyer, and D. Marx, Phys. Rev. Lett. 105, 146405 (2010).

34 A. Janotti, C. Franchini, J. B. Varley, G. Kresse, and C. G. Van de Walle, Dual behavior of excess electrons in rutile $\mathrm{TiO}_{2}$, Vol. 7 (Wiley-Blackwell, 2013) pp. 199-203.

35 H. Ibach and D. L. Mills, Electron Energy Loss Spectroscopy and Surface Vibrations, edited by Academic Press (Academic Press, New York, 1982).

36 L. Vattuone, L. Savio, and M. Rocca, "Surface science techniques," (Springer Verlag, 2013) Chap. High Resolution Electron Energy Loss Spectroscopy (HREELS): A sensitive and versatile surface tool, pp. 499-529.

37 H. Ibach, Phys. Rev. Lett. 24, 1416 (1970).

38 P. Lambin, L. Henrard, P. Thiry, C. Silien, and J. P. Vigneron, J. Electron Spectrosc. Relat. Phenom. 129, 281 (2003).

39 Y. Goldstein, A. Many, and I. Wagner, Surf. Sci. 98, 599 (1980).

40 A. Many, I. Wagner, A. Rosenthal, J. I. Gersten, and Y. Goldstein, Phys. Rev. Lett. 46, 1648 (1981).

41 Y. Wang, B. Meyer, X. Yin, M. Kunat, D. Langenberg, F. Traeger, A. Birkner, and C. Wöll, Phys. Rev. Lett. 95, 266104 (2005).

${ }^{42}$ H. Qiu, B. Meyer, Y. Wang, and C. Wöll, Phys. Rev. Lett. 101, 236401 (2008).

43 B. N. J. Persson and J. E. Demuth, Phys. Rev. B 30, 5968 (1984).

44 J. Stroscio and W. Ho, Phys. Rev. Lett. 54, 1573 (1985).

45 J. A. Stroscio and W. Ho, Phys. Rev. B 36, 9736 (1987).
46 A. Ritz and Lüth, Phys. Rev. Lett. 52, 1242 (1984).

47 T. Inaoka and D. M. Newns, Surf. Sci. 186, 290 (1987).

48 Y. Chen, J. C. Hermanson, and G. J. Lapeyre, Phys. Rev. B 39, 12682 (1989).

49 R. Matz and H. Lüth, Phys. Rev. Lett. 46, 500 (1981).

${ }^{50}$ M. Noguchi, K. Hirakawa, and T. Ikoma, Surf. Sci. 271, 260 (1992).

51 V. M. Polaakov, A. Elbe, J. Wu, G. J. Lapeyre, and J. A. Schaefer, Phys. Rev. B 54, 2010 (1996).

52 A. A. Lucas and M. Sunjić, Phys. Rev. Lett. 26, 229 (1971).

53 A. A. Lucas and M. Sunjić, Prog. Surf. Sci. 2, 75 (1972).

54 E. Evans and D. L. Mills, Phys. Rev. B 5, 4126 (1972).

55 E. Evans and D. L. Mills, Phys. Rev. B 7, 853 (1973).

56 D. L. Mills, Surf. Sci. 48, 59 (1975).

57 A. A. Lucas and J. P. Vigneron, Solid. Stat. Comm. 49, 327 (1984).

58 P. Lambin, J. P. Vigneron, and A. A. Lucas, Phys. Rev. B 32, 8203 (1985).

59 P. A. Cox, R. G. Edgell, S. Eriksen, and W. R. Flavell, J. Electron. Spectrosc. and Relat. Phenom. 39, 117 (1986).

${ }^{60}$ L. Kesmodel, J. Gates, and Y. Chung, Phys. Rev. B 23, 489 (1981).

61 M. A. Henderson, Surf. Sci. 355, 151 (1996).

${ }^{62}$ M. A. Henderson, W. S. Epling, C. L. Perkins, C. H. F. Peden, and U. Diebold, J. Phys. Chem. B 103, 5328 (1999).

${ }^{63}$ M. A. Henderson, W. S. Epling, C. H. F. Peden, and C. L. Perkins, J. Phys. Chem. B 107, 534 (2003).

64 M. A. Henderson, M. Shen, Z.-T. Wang, and I. Lyubinetsky, J. Phys. Chem. C 117, 5774 (2013).

${ }^{65}$ H. Noei, L. Jin, H. Qiu, M. Xu, Y. Gao, J. Zhao, M. Kauer, C. Wöll, M. Muhler, and Y. Wang, Phys. Status Solidi B 250, 1204 (2013).

66 F. Gervais and J. F. Baumard, Solid State Commun. 21, 861 (1977).

67 J. F. Baumard and F. Gervais, Phys. Rev. B 15, 2316 (1977).

68 P. A. Thiry, M. Liehr, J.-J. Pireaux, and R. Caudano, Phys. Scripta 35, 368 (1987).

69 J. Li, Origin, location and transport of excess charges in titanium dioxide, Ph.D. thesis, Pierre and Marie Curie University, France (2016).

${ }^{70}$ W. Schaich, Surf. Sci. 122, 175 (1982).

71 See Supplemental Material at URLXXX for a description (i) of the dielectric theory of EELS of uniaxial material and graded interface (Sect. S1), (ii) of the effect of anisotropy (Sect. S2), (iii) of the analytic equation of plasmon-induced broadening of the quasi-elastic peak (Sect. S3) and (iv) for complementary figures and tables (Sect. S4).

72 The notations $\|, \perp$ of the components of the dielectric tensor and of the scattering geometry have different meanings.

73 P. A. Cox, W. R. Flavell, A. A. Williams, and R. G. Egdell, Surf. Sci. 152-153, 784 (1985).

74 R. Piessens, E. deDoncker Kapenga, C. Uberhuber, and D. Kahaner, Quadpack: a Subroutine Package for Automatic Integration, edited by Springer and Verlag, Series in Computational Mathematics v.1 (1983).

75 H. Ibach, Electron Energy Loss Spectrometers: The technology of high performance (Springer-Verlag, 1991).

76 E. D. Palik, Handbook of Optical Constants of Solids, Vol. 1-3 (Academic Press, 1985). 
77 F. Gervais and B. Piriou, Phys. Rev. B 10, 1642 (1974).

78 F. Gervais, Materials Science And Engineering R-Reports 39, 29 (2002).

79 D. M. Eagles, J. Phys. Chem. Solids 25, 1243 (1964).

80 J. G. Traylor, H. G. Smith, R. M. Nicklow, and M. K. Wilkinson, Phys. Rev. B 3, 3457 (1971).

81 C. Lee, P. Ghosez, and X. Gonze, Phys. Rev. B 50, 13379 (1994).

82 P. Mitev, K. Hermansson, B. Montanari, and K. Refson, Phys. Rev. B 81, 134303 (2010).

83 M. Dou and C. Persson, J. Appl. Phys. 113, 083703 (2013).

84 Private communication.

85 R. Betsch, H. Park, and W. White, Mater. Res. Bull. 26, 613 (1991).

${ }^{86}$ N. W. Aschcroft and N. D. Mermin, Physique des solides (EDP Sciences, 2002).

87 G. Samara and P. Peercy, Phys. Rev. B 7, 1131 (1973).

88 J. Pascual, J. Camassel, and H. Mathieu, Phys. Rev. B 18, 5606 (1978).

89 L. Chiodo, J. M. García-Lastra, A. Iacomino, S. Ossicini, J. Zhao, H. Petek, and A. Rubio, Phys. Rev. B 82, 045207 (2010).

90 N. Vast, L. Reining, V. Olevano, P. Schattschneider, and B. Jouffrey, Phys. Rev. Lett. 88, 037601 (2002).

91 I. G. Gurtubay, W. Ku, J. M. Pitarke, A. G. Eguiluz, B. C. Larson, J. Tischler, and P. Zschack, Phys. Rev. B 70, 201201 (2004).

92 J. Leng, J. Opsala, H. Chua, M. Senkoa, and D. Aspnes, Thin Solid Films 313-314, 132 (1998).

93 A. Djurisic, Y. Chan, and E. Li, Materials Science And Engineering R-Reports 38, 237 (2002).

94 J. T. Devreese and A. S. Alexandrov, Reports on Progress in Physics 72, 066501 (2009).

95 A. S. Alexandrox and J. Devreese, Advances in polaron physics (Spinger, 2010).

96 K. Onda, B. Li, and H. Petek, Phys. Rev. B 70, 0454415 (2004).

97 S. Wendt, P. T. Sprunger, E. Lira, G. K. H. Madsen, Z. Li, J. O. Hansen, J. Matthiesen, A. Blekinge-Rasmussen, E. Laegsgaard, B. Hammer, and F. Besenbacher, Science 320, 1755 (2008).

${ }^{98}$ K. Mitsuhara, H. Okumura, A. Visikovskiy, M. Takizawa, and Y. Kido, J. Chem. Phys. 136, 124707 (2012).

99 X. Mao, X. Lang, Z. Wang, Q. Hao, B. Wen, Z. Ren, D. Dai, C. Zhou, L. M. Liu, and X. Yang, J. Phys. Chem. Lett. 4, 3839 (2013).

100 T. Minato, Y. Sainoo, Y. Kim, H. S. Kato, K. Aika, M. Kawai, J. Zhao, H. Petek, T. Huang, W. He, W. Bing, W. Zhuo, Y. Zhao, J. Yang, and J. G. Hou, J. Chem. Phys. 130, 124502 (2009).

101 S. Moser, L. Moreschini, J. Jaćimović, O. S. Barišić, H. Berger, A. Magrez, Y. J. Chang, K. S. Kim, A. Bostwick, E. Rotenberg, L. Forró, and M. Grioni, Phys. Rev. Lett. 110, 196403 (2013).

102 C. Verdi, F. Caruso, and F. Giustino, Nat. Comm. 8, 15769 (2017).

103 T. He, Phys. Rev. B 51, 16689 (1995).

104 P. G. Moses, A. Janotti, C. Franchini, G. Kresse, and C. G. Van de Walle, J. Appl. Phys. 119, 181503 (2016), http://dx.doi.org/10.1063/1.4948239.

105 R. R. Hasiguti and E. Yagi, Phys. Rev. B 49, 7251 (1994).

106 E. Hendry, M. Koeberg, J. Pijpers, and M. Bonn, Phys. Rev. B 75, 233202 (2007).

107 F. Herklotz, E. Lavrov, and J. Weber, Phys. Rev. B 83, 235202 (2011).

108 J. D. Jackson, Classical Electrodynamics, edited by Wiley (Wiley \& Sons, New York, 1975).

109 R. Lazzari, J. Li, and J. Jupille, Rev. Sci. Instrum. 86, 013906 (2015).

110 J. I. Gersten, Surf. Sci. 92, 579 (1980). 


\title{
Supplemental Material: \\ Dielectric study of the interplay between charge carriers and electron energy losses in reduced titanium dioxide
}

\author{
Rémi Lazzari,* Jingfeng Li, and Jacques Jupille \\ CNRS, Sorbonne Université, Institut des NanoSciences de Paris, \\ UMR 7588, 4 Place Jussieu, F-75005 Paris, France
}

(Dated: August 17, 2018)

\section{SI. DIELECTRIC THEORY OF ELECTRON ENERGY LOSS SPECTROSCOPY IN REFLECTION: UNIAXIAL DIELECTRIC FUNCTION AND STRATIFIED MEDIUM}

For an uniaxial material like tetragonal $\mathrm{TiO}_{2}$ rutile, the dielectric tensor is anisotropic but diagonal in the main crystallographic axis with two principal components, $\epsilon_{\perp}^{a}(\omega)$ perpendicular to the c-axis (or parallel to the a-axis) and $\epsilon_{\|}^{c}(\omega)$ parallel to the c-axis (or perpendicular to the a-axis). For such an uniaxial material, the loss function depends on the scattering geometry ${ }^{1}$. Assuming that the $\mathrm{c}$-axis lies in the $(x, z)$-plane at an angle $\theta$ from the surface normal $z, \xi\left(\mathbf{k}_{\|}, \omega\right)$ is given by ${ }^{1,2}$

$$
\xi\left(\mathbf{k}_{\|}, \omega\right)=\frac{\epsilon_{z z}(\omega)}{k_{\|}} \sqrt{\frac{\epsilon_{\|}^{c}(\omega) \epsilon_{\perp}^{a}(\omega)}{\epsilon_{z z}^{2}(\omega)} k_{x}^{2}+\frac{\epsilon_{\perp}^{a}(\omega)}{\epsilon_{z z}(\omega)} k_{y}^{2}},
$$

where $\epsilon_{z z}(\omega)=\epsilon_{\|}^{c}(\omega) \cos ^{2}(\theta)+\epsilon_{\perp}^{a}(\omega) \sin ^{2}(\theta)$ is the component of the dielectric tensor normal to the surface and $\left(k_{x}, k_{y}\right)$ are the components of the electron wave vector transfer parallel to the surface. At $\mathrm{TiO}_{2}(110)$ surface ${ }^{3,4}$, the c-axis lies in the surface plane along the $x$-axis $\left(\theta=90^{\circ}\right)$ and $\xi\left(\mathbf{k}_{\|}, \omega\right)=1 / k_{\|} \sqrt{\epsilon_{\|}^{c}(\omega) \epsilon_{\perp}^{a}(\omega) k_{x}^{2}+\epsilon_{\perp}^{a}(\omega)^{2} k_{y}^{2}}$. The cumbersome integration in Eq. 1 of the paper over the detector acceptance and incident beam divergence can be avoided in two extreme cases where $\xi\left(\mathbf{k}_{\|}\right)$becomes $k_{\|}$-independent. Either the dominant scattering is in the $(y, z)$-plane, i.e. $k_{x} \simeq 0$ leading to $\xi\left(\mathbf{k}_{\|}, \omega\right) \simeq \epsilon_{\perp}^{a}(\omega)$ as in experiments of Refs. 5 and 6 . Or it is in the $(x, z)$-plane, i.e. $k_{y} \simeq 0$ and $\xi\left(\mathbf{k}_{\|}, \omega\right) \simeq \sqrt{\epsilon_{\perp}^{a}(\omega) \epsilon_{\|}^{c}(\omega)}$; in this case, the interpretation is less straightforward as losses result from a geometric mean of parallel and perpendicular dielectric behaviors.

Lambin et $a .^{7}$ derived also the loss function for a stratified medium having a given profile of isotropic dielectric function $\epsilon(\omega, z)$. They found a recursive formula which relates the value of the effective dielectric function $\xi\left(\omega, z_{i-1}\right)$ at the lower end of a layer of thickness $d_{i}$ and its dielectric constant $\epsilon\left(\omega, z_{i}\right)$ to the value of the function at the upper end of the layer $\xi\left(\omega, z_{i}\right)$ :

$\xi\left(\omega, z_{i-1}\right)=\epsilon\left(\omega, z_{i}\right) \operatorname{coth}\left(k_{\|} d_{i}\right)-\frac{\left[\epsilon\left(\omega, z_{i}\right) / \sinh \left(k_{\|} d_{i}\right)\right]^{2}}{\epsilon\left(\omega, z_{i}\right) \operatorname{coth}\left(k_{\|} d_{i}\right)+\xi\left(\omega, z_{i}\right)}$
The starting point is given by the substrate dielectric function $\xi\left(\omega, z_{\infty}\right)=\epsilon_{S}(\omega)$. The classical loss probability $P_{c l}\left(\mathbf{k}_{I}, \omega\right)$ (Eq. 1 of the paper) is then calculated from the surface effective dielectric function, i.e. $\xi\left(\omega, z_{0}\right)$ which can be recasted in to a continued fraction expansion $^{7}$ from Eq. S2. Through recursivity and slicing, it is therefore possible to describe any profile of dielectric function as done in the paper. The generalization to a stack of anisotropic media is slightly more complex ${ }^{2}$ but fortunately with a dominant scattering normal to the c-axis of $\mathrm{TiO}_{2}$, the above formula is still valid but with $\epsilon_{\perp}^{a}(\omega)$.

\section{SII. EFFECT OF DIELECTRIC ANISOTROPY ON (HR)EELS SPECTRUM}

Figure $\mathrm{S} 1$ shows simulated spectra at low $\left[E_{I}=8 \mathrm{eV}\right.$, Fig. S1(a) $]$ and high $\left[E_{I}=38 \mathrm{eV}\right.$, Fig. S1(b)] impact energies for the two main orientations of the c-axis leading to different expression of the effective dielectric function $\xi(\omega)$ (see Sec. SI). This latter amounts to $\xi(\omega)=\epsilon_{\perp}^{a}(\omega)$ [respectively $\left.\xi(\omega)=\sqrt{\epsilon_{\|}^{c}(\omega) \epsilon_{\perp}^{a}(\omega)}\right]$ for and incident plane perpendicular (respectively parallel) to the c-axis. Simulations are also compared to a fictitious material having a effective dielectric function equal to $\xi(\omega)=\epsilon_{\|}^{c}(\omega)$.

When the c-axis is perpendicular to the incident plane, the loss spectrum contains only contributions from the $E_{u}$-modes and the high energy surface phonon $\omega_{s p h, 3}=$ $95.6 \mathrm{meV}$ falls just below the longitudinal $E_{u}$ phonon at $\omega_{L O}=102.9 \mathrm{meV}$. The fictitious spectra with $\epsilon_{\|}^{c}(\omega)$ is dominated by the $A_{2 u}$ mode with a surface peak at $\omega_{s p h, \|}=92.4 \mathrm{meV}$. When the c-axis is parallel to the incident beam, the symmetry assignment is less rigorous since $\xi(\omega)=\sqrt{\epsilon_{\|}^{c}(\omega) \epsilon_{\perp}^{a}(\omega)}$, but the low energy peaks are clearly of $E_{u}$-type while the high energy one at $\omega_{s p h, 3}=93.9 \mathrm{meV}$ combines the high energy $E_{u}$ and $A_{2 u}$ longitudinal modes. The latter screens the low energy $E_{u}$ surface modes which are much weaker in intensity than along the other orientation. The discussion on phonon shifts and changes of intensity are restricted in the paper to the $\xi(\omega)=\epsilon_{\perp}^{a}(\omega)$ case since, due to the fortuitous near degeneracy of the high energy $A_{2 u}$ and $E_{u}$ longitudinal modes ${ }^{3,4}$, the differences between the two 

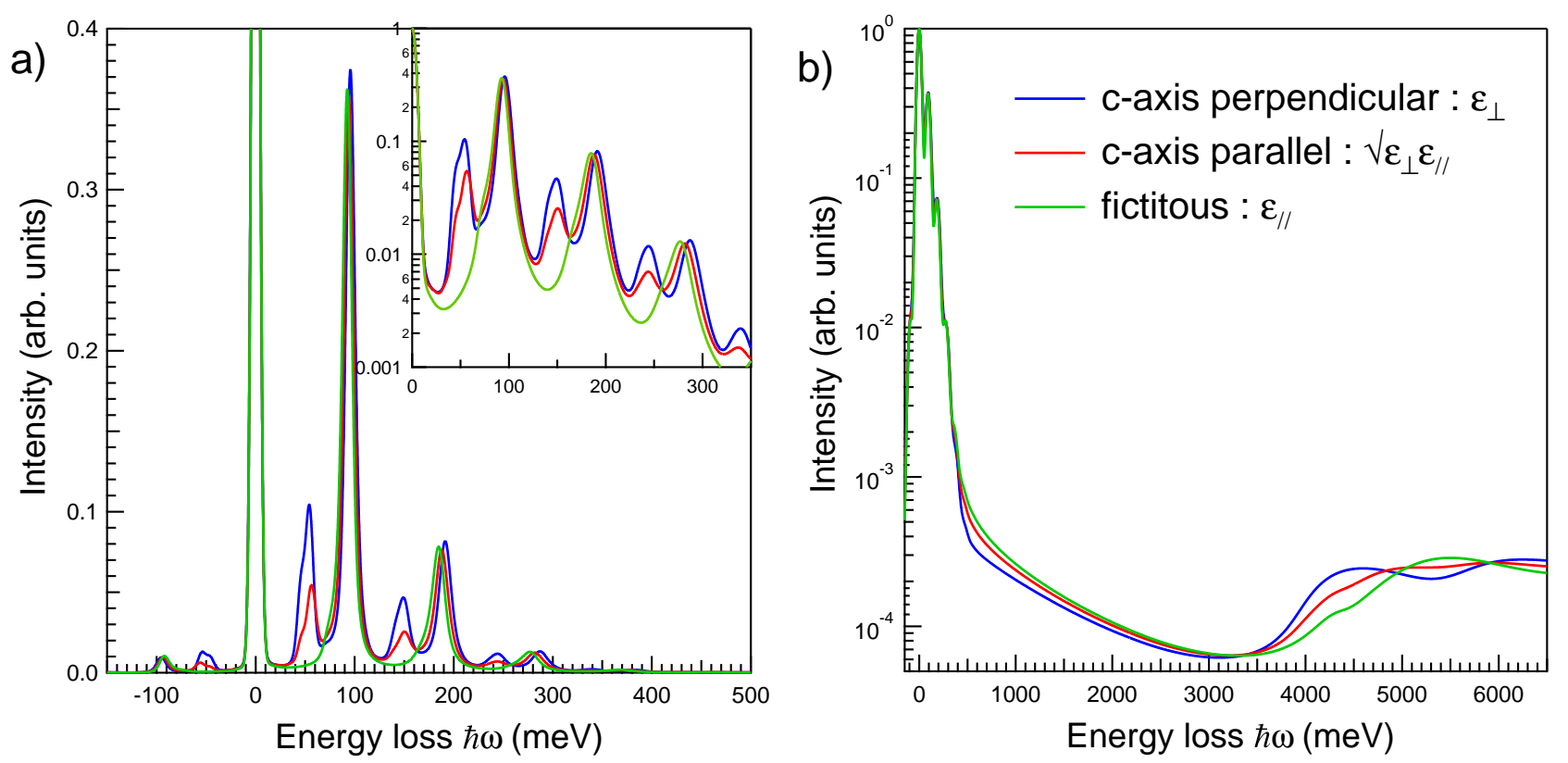

FIG. S1. Effect of the uniaxial anisotropy of $\mathrm{TiO}_{2}$ on simulated (a) HREELS $\left(E_{I}=8 \mathrm{eV} ; \Delta_{a}=8\right.$ meV) phonon spectrum, and (b) EELS $\left(E_{I}=38 \mathrm{eV} ; \Delta_{a}=50 \mathrm{meV}\right)$ interband transitions. Blue line: c-axis perpendicular to beam; red line: c-axis parallel to beam; green line: fictitious material having a $\epsilon_{\|}^{c}$ dielectric function. Spectra have been normalized to the elastic peak intensity and no band-gap state feature has been accounted for. The inset shows a zoom of the spectrum on a logarithmic scale.

main c-axis orientations are minor for the high energy surface phonon $\omega_{s p h, 3}$. Nevertheless, tiny shifts and differences are amplified in the multiple and combination losses that structure EELS spectra ${ }^{8,9}$ and therefore easier to detect despite a modest experimental resolution [inset of Fig. S1(a)]. Finally, regarding band-to-band transitions probed at high energy [Fig. S1(b)], the various orientations slightly differ through anisotropy (Fig. 1 of the paper). The $\epsilon_{\perp}^{a}(\omega)$-orientation offers the lowest background at the location of the BGS.

\section{SIII. ANALYTIC DESCRIPTION OF PLASMON-INDUCED BROADENING OF THE QUASI-ELASTIC PEAK}

Starting from a $t$-expansion of the Poisson distribution of multiple losses (Eqs. 3-6 of the aricle), Bo Persson and Demuth ${ }^{10}$ derived an elegant analytic formula for the variance of the broadening of the elastic peak due to bulk free carriers described by a Drude term:

$$
\begin{aligned}
& \Delta_{n}^{2}=\frac{C}{4} \pi^{2} \omega_{s p}^{2}\left[\frac{2}{e^{\hbar \omega_{s p} / k_{B} T}-1}+1\right] \\
& \text { with } \quad C=\frac{4}{\pi} \frac{1}{\epsilon(0)+1} \frac{1}{\cos ^{2} \Theta_{I}} \frac{1}{k_{I} a_{0}},
\end{aligned}
$$

where $a_{0}=4 \pi \epsilon_{0} \hbar^{2} / m e^{2}$ is the Bohr radius and the other parameters are defined in the paper. By adding an appa- ratus function $\Delta_{a}^{2}$, the evolution of the quasi-elastic fullwidth at half-maximum $\left(\mathrm{FWHM}=2 \sqrt{2 \ln 2} \sqrt{\Delta_{n}^{2}+\Delta_{a}^{2}}\right)$ can be obtained either as a function of carrier concentration at fixed temperature [Fig. 4(a) of the paper, dotted line] or vice-versa (Fig. 5 of the paper, dotted lines). For instance, Eq. S3 has been used to determine the ionization energy of hydrogen-doped $\mathrm{ZnO}^{11}$ from the temperature dependence of the quasi-elastic peak broadening. But the rutile elastic peak is much less sensitive to broadening because of its much higher static dielectric constant and effective mass (smaller $\omega_{s p}$ ) than $\mathrm{ZnO}$. A temperature dependence is also expected from Eq. S3 for rutile [Fig. 5(a) of the paper, red and violet dotted lines] on the basis of a carrier concentration of $n=10^{18-19} \mathrm{~cm}^{-3}$ (Refs. 12-14) and of an effective mass of $m^{*} \simeq 10$ (Refs. 15 and 16). Useful to pinpoint the main phenomena, the approach of Bo Persson and Demuth is valid only for infinitesimal $\Gamma_{P}$ and $\omega_{s p}$, a fact which is not overlooked in the analysis of Ref. 11. The analytic equation Eq. S3 does not even reproduce the behavior at very small damping (Fig. 4 of the paper, black dotted vs black full line) since the underlying limit $\omega_{s p} \rightarrow 0$ is not reached; it also overestimates the temperature dependence that is determined in the paper with full numerical simulations (Fig. 5 of the paper). 


\section{SIV. COMPLEMENTARY TABLES AND FIGURES}

* Corresponding author :remi.lazzari@insp.jussieu.fr

1 A. A. Lucas and J. P. Vigneron, Solid. Stat. Comm. 49, 327 (1984).

2 P. Lambin, L. Henrard, P. Thiry, C. Silien, and J. P. Vigneron, J. Electron Spectrosc. Relat. Phenom. 129, 281 (2003).

3 P. A. Cox, R. G. Edgell, S. Eriksen, and W. R. Flavell, J. Electron. Spectrosc. and Relat. Phenom. 39, 117 (1986).

4 S. Eriksen and R. G. Egdell, Surf. Sci. 180, 263 (1987).

5 J. Li, R. Lazzari, S. Chenot, and J. Jupille, Phys. Rev. B Rapid Comm. 97, 041403(R) (2018).

6 J. Li, Origin, location and transport of excess charges in titanium dioxide, Ph.D. thesis, Pierre and Marie Curie University, France (2016).

7 P. Lambin, J. P. Vigneron, and A. A. Lucas, Phys. Rev. B 32, 8203 (1985).

${ }^{8}$ G. Rocker, J. A. Schaefer, and W. Göpel, Phys. Rev. B 30, 3704 (1984).
9 R. Lazzari, J. Li, and J. Jupille, Rev. Sci. Instrum. 86, 013906 (2015).

10 B. N. J. Persson and J. E. Demuth, Phys. Rev. B 30, 5968 (1984).

11 H. Qiu, B. Meyer, Y. Wang, and C. Wöll, Phys. Rev. Lett. 101, 236401 (2008).

12 J. F. Baumard and F. Gervais, Phys. Rev. B 15, 2316 (1977).

13 T. Bak, J. Nowotny, M. Rekas, and C. Sorrell, J. Phys. Chem. Solids 64, 1043 (2003).

14 U. Diebold, Surf. Sci. Rep. 48, 53 (2003).

15 E. Hendry, F. Wang, J. Shan, T. F. Heinz, and M. Bonn, Phys. Rev. B 69, 081101(R) (2004).

16 E. Yagi, R. R. Hasiguti, and M. Aono, Phys. Rev. B 54, 7945 (1996).

17 D. Schöche, T. Hofmann, R. Korlacki, T. Tiwald, and M. Schubert, J. Appl. Phys. 113, 164102 (2013).

18 D. M. Eagles, J. Phys. Chem. Solids 25, 1243 (1964).

19 E. D. Palik, Handbook of Optical Constants of Solids, Vol. 1-3 (Academic Press, 1985). 


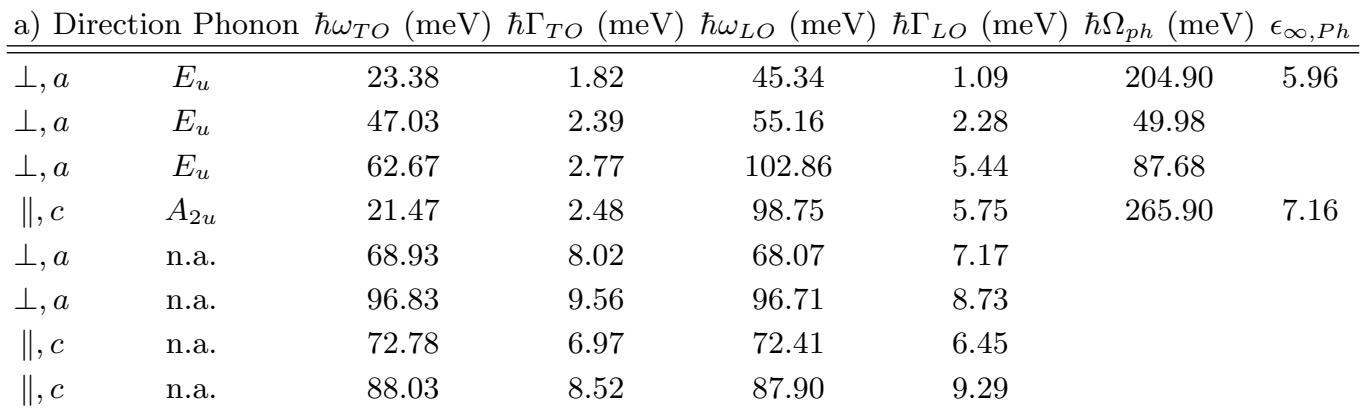

\begin{tabular}{ccccccc} 
b) Direction $\hbar C\left(\mathrm{meV}^{\mu}\right) \times 10^{-3}$ & $\Phi(\mathrm{deg})$ & $\hbar \omega(\mathrm{meV})$ & $\hbar \Gamma(\mathrm{meV})$ & $\mu$ & $\epsilon_{\infty, I b}$ \\
\hline \hline$\|, c$ & 2.5 & 0 & 4.09 & 0.28 & $0.8-5.26$ \\
$\|, c$ & 0.37 & 0 & 4.41 & 0.18 & 0.7 & \\
$\|, c$ & 5.2 & 0 & 6.63 & 1.15 & 1.0 \\
$\perp, a$ & 0.28 & 0 & 3.92 & 0.21 & $0.5-6.86$ \\
$\perp, a$ & 1.4 & 0 & 5.41 & 0.44 & 1.0 \\
$\perp, a$ & 19.3 & 0 & 7.52 & 1.46 & 1.3
\end{tabular}

\begin{tabular}{|c|c|c|c|}
\hline c) Direction & $\omega_{s p h, 1}(\mathrm{meV})$ & $\omega_{s p h, 2}(\mathrm{meV})$ & $\omega_{s p h, 3}(\mathrm{meV})$ \\
\hline $\operatorname{Im}\left[-1 /\left(1+\epsilon_{\perp}^{a}\right)\right]$ & 45.3 & 54.6 & 95.4 \\
\hline $\operatorname{Im} \mid-1 /\left(1+\sqrt{\epsilon_{\|}^{c} \epsilon_{\perp}^{a}}\right.$ & 45.6 & 55.4 & 93.8 \\
\hline
\end{tabular}

TABLE S1. (a) Parameters of the factorized dielectric function $\epsilon_{P h}(\omega)$ (Eq. 8 of the paper) of the infrared active optical phonons $3 \mathrm{E}_{u}+\mathrm{A}_{2 u}$ (from Ref. 17). Values of symmetry forbidden not assigned (n.a.) minor modes are also given. Error bars on frequencies and dampings are below $0.1 \mathrm{meV}$ and $0.6 \mathrm{meV}$ (respectively $1.6 \mathrm{meV}$ and $0.6 \mathrm{meV}$ ), for the symmetry allowed modes (respectively the additional ones). The oscillator strengths $\Omega_{p h}$ of the unfactorized form of the dielectric function (see Eq. 15 of the paper) of Ref. 18 are also given as well as the prefactor $\epsilon_{\infty, P h}$. The static values are: $\epsilon_{\|}^{c}(0)=153$ and $\epsilon_{\perp}^{a}(0)=84.7$. (b) Critical point transition parameters (Eq. 10 of the paper) used in the fit of the UV-visible part of the dielectric function $\epsilon_{I b}(\omega)$ of Ref. 19. Negative values for $\epsilon_{\infty, I b}$ are counterbalanced by $\epsilon_{\infty, P h}$ at $\omega \rightarrow+\infty$. (c) Positions of the surface phonon modes as given by the maxima of the loss function.

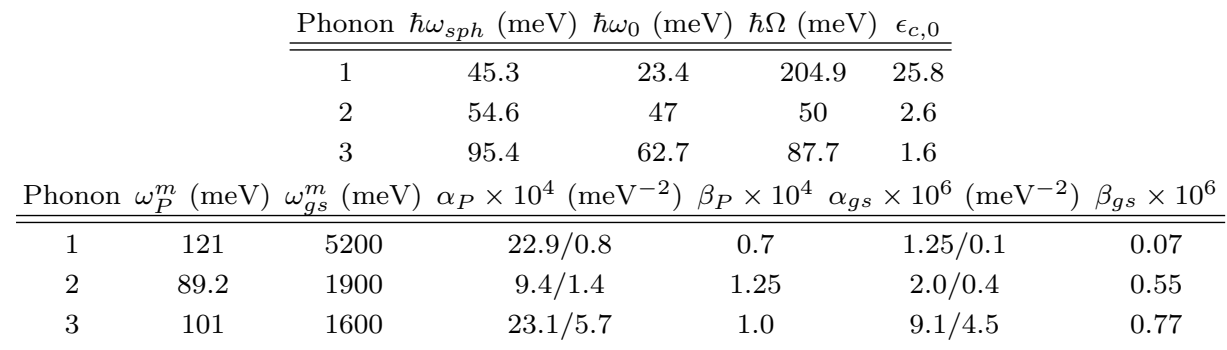

TABLE S2. Screening effect of surface phonons by plasmon and band gap states: surface frequency $\omega_{s p h}$, transverse frequency $\omega_{0}=\omega_{T O}$, oscillator strength $\Omega$ from decomposition of Ref. 18, screening value $\epsilon_{c, 0}$, maximum value for linearity $\omega_{P}^{m}=$ $\omega_{0} \sqrt{1+\epsilon_{c, 0}}$ and $\omega_{g s}^{m}=\omega_{g s} \sqrt{1+\epsilon_{c, 0}}$, slopes $\alpha_{P}, \alpha_{g s}$ and $\beta_{P}, \beta_{g s}$ as a function of $\omega_{P}^{2}$ and $\Omega_{g s}^{2}$, respectively (see paper for definition). 

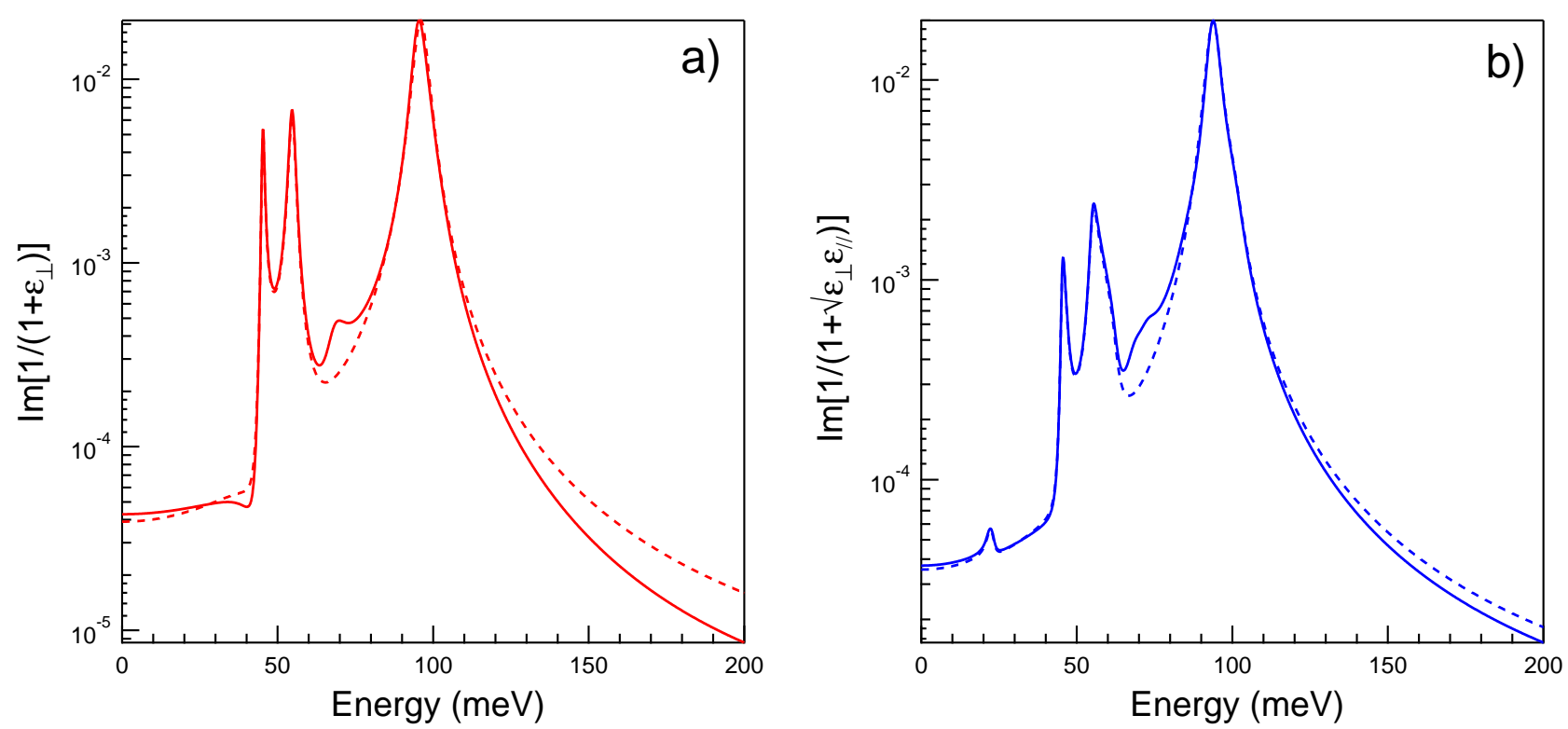

FIG. S2. Phonon loss functions (a) perpendicular $\operatorname{Im}\left[-\frac{1}{1+\epsilon_{\perp}^{a}(\omega)}\right]$ and (b) parallel $\operatorname{Im}\left[-\frac{1}{1+\sqrt{\left.\epsilon_{\|}^{c}(\omega) \epsilon_{\perp}^{a}(\omega)\right)}}\right]$ to the c-axis with (full lines) and without (dotted lines) additional symmetry forbidden modes.

\section{a) Parallel}
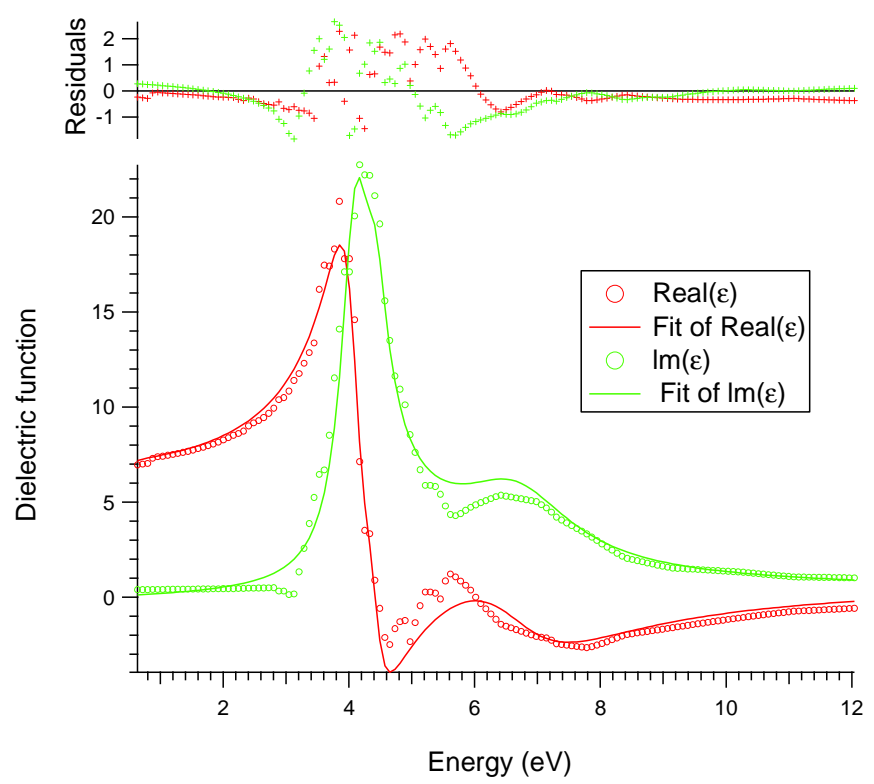

b) Perpendicular
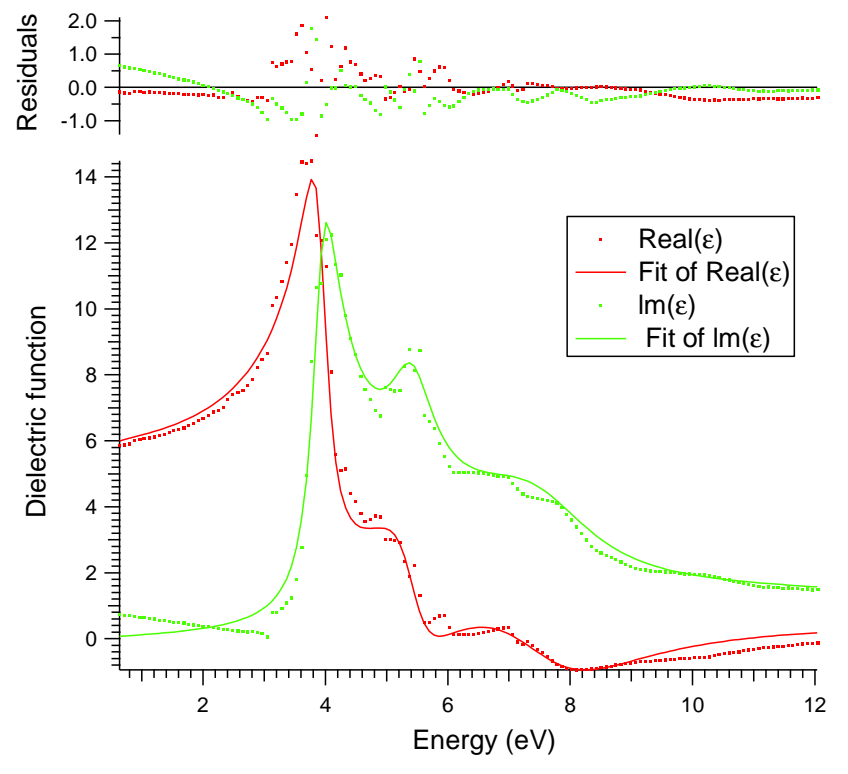

FIG. S3. Fit of the UV-visible dielectric function $(a) \epsilon_{\|, I b}^{c}(\omega)$ and $(b) \epsilon_{\perp, I b}^{a}(\omega)$ of $\mathrm{TiO}_{2}$ from dielectric database of Ref. 19. The analysis was performed with three critical transition points as explained in the paper. 